\title{
Correlating Structure and Detection Properties in HgTe Nanocrystal Films
}

Sang-Soo Chee ${ }^{1,2}$, Charlie Gréboval ${ }^{1}$, Debora Vale Magalhaes ${ }^{3,4}$, Julien Ramade ${ }^{3,4}$, Audrey Chu ${ }^{1}$, Junling Qu ${ }^{1}$, Prachi Rastogi ${ }^{1}$, Adrien Khalili ${ }^{1}$, Tung Huu Dang ${ }^{1,5}$, Corentin Dabard ${ }^{1}$, Yoann Prado ${ }^{1}$, Gilles Patriarche ${ }^{6}$, Julien Chaste ${ }^{6}$, Michael Rosticher ${ }^{5}$, Sara Bals ${ }^{3,4}$, Christophe Delerue ${ }^{7}$, Emmanuel Lhuillier ${ }^{1^{*}}$

${ }^{1}$ Sorbonne Université, CNRS, Institut des NanoSciences de Paris, 4 place Jussieu, 75005 Paris, France.

2 Nanomaterials and Nanotechnology Center, Korea Institute of Ceramic Engineering and Technology (KICET), 101 Soho-ro, 52851 Jinju-si, Republic of Korea

${ }^{3}$ Electron Microscopy for Materials Science, University of Antwerp, Groenenborgerlaan 171, B2020 Antwerp, Belgium.

${ }^{4}$ NANOlab Center of Excellence, University of Antwerp, Belgium

${ }^{5}$ Laboratoire de Physique de l'Ecole normale supérieure, ENS, Université PSL, CNRS, Sorbonne Université, Université Paris-Diderot, Sorbonne Paris Cité, Paris, France Paris.

${ }^{6}$ Centre de Nanosciences et de Nanotechnologies, CNRS, Université Paris-Saclay, C2N, Palaiseau 2110, France.

7 Univ. Lille, CNRS, Centrale Lille, Univ. Polytechnique Hauts-de-France, Junia, UMR 8520 IEMN, F-59000 Lille, France

To whom correspondence should be sent: el@insp.upmc.fr

\section{Table of content}

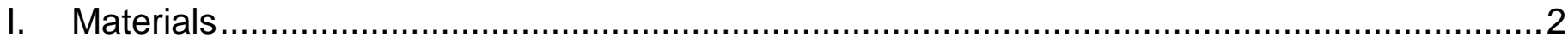

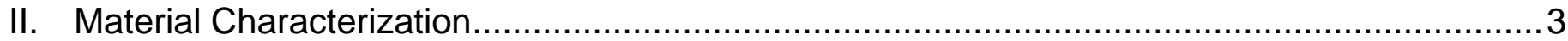

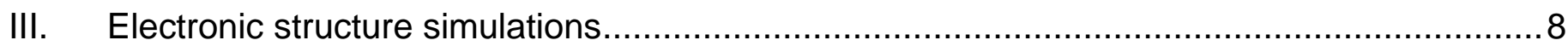

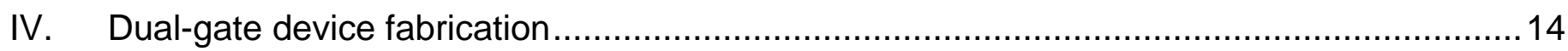

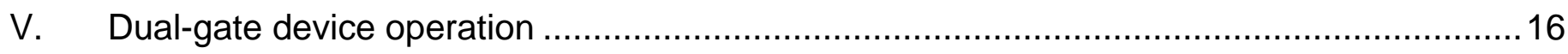

VI. Dual-gate device performances for photodetection............................................. 17

VII. Comparison of dual gate device for SWIR sensing with state-of-the-art devices ...............20

VIII. Comparison HgTe CQD-based vertical geometry photodiode .....................................20

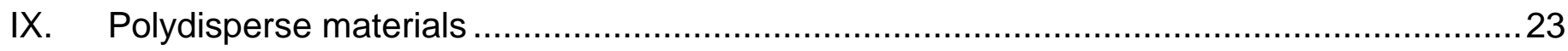

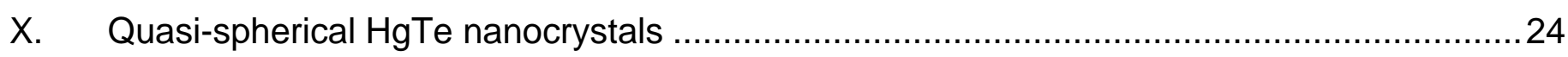

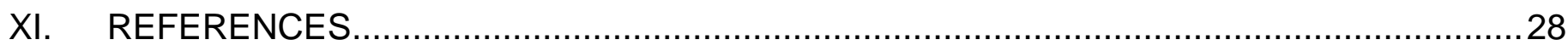




\section{Materials}

Chemicals for Colloidal Qantum Dot (CQD) synthesis. Mercury chloride $\left(\mathrm{HgCl}_{2}\right.$, Strem Chemicals, $99 \%$ ), Mercury salts are toxic, handle them with great care, tellurium powder (Te, Sigma-Aldrich, 99.99\%), trioctylphosphine (TOP, Alfa-Aesar, 90\%), silver Nitrate $\left(\mathrm{AgNO}_{3}\right.$, Prolabo, 99.5\%), oleylamine (OLA, Acros, 80-90\%), dodecanethiol (DDT, Sigma-Aldrich, 98\%), oleic acid (OA, Afla Aesar, 90\%), 1,2,ethanedithiol (EDT, Fluka, 98.0\%), mercaptoalcohol (MpOH, Merck, $>99 \%$ ), chloroform (VWR), ethanol absolute anhydrous (VWR), methanol (Carlo Erba, 99.8\%), octane (Carlo erba, 99\%), acetonitrile (VWR, 99.9\%) acetone (VWR), n-hexane (VWR, 99\%), toluene (Carlo Erba, 99.3\%), N,N-dimethylformamide (DMF, Sigma Aldrich), chlorobenzene (VWR), Methylisobutylketone (MIBK, VWR, $>98.5 \%$ ). All chemicals are used as received.

$1 \mathrm{M}$ TOP:Te precursor: In a three-neck flask, $2.54 \mathrm{~g}$ of Te powder are mixed in $20 \mathrm{~mL}$ of TOP. The flask is kept under vacuum at room temperature for $5 \mathrm{~min}$ and then is heated up to $100^{\circ} \mathrm{C}$ and kept under vacuum for 20 more minutes. The atmosphere is changed to $\mathrm{N}_{2}$ and the temperature is raised to $275^{\circ} \mathrm{C}$. The solution is stirred until a clear orange coloration is obtained. Then the flask is cooled down and the color switches to yellow. The flask is degassed again for $10 \mathrm{~min}$ at room temperature. This solution is finally transferred to an $\mathrm{N}_{2}$-filled glovebox for storage.

HgTe tripod synthesis: In a $100 \mathrm{~mL}$ three neck flask, $543 \mathrm{mg}$ of $\mathrm{HgCl}_{2}$ and $50 \mathrm{~mL}$ of oleylamine are degassed under vacuum at $110^{\circ} \mathrm{C}$. Meanwhile, $2 \mathrm{~mL}$ of TOP:Te $(1 \mathrm{M})$ are extracted from the glove box and mixed with $8 \mathrm{~mL}$ of oleylamine. After the atmosphere is switched to $\mathrm{N}_{2}$ and the temperature stabilized at $82{ }^{\circ} \mathrm{C}$, the TOP:Te solution is quickly injected. After $2 \mathrm{~min} 30 \mathrm{~s}, 10 \mathrm{~mL}$ of a mixture of DDT in toluene (10\% of DDT) is injected and a water bath is used to quickly decrease quickly the temperature. The content of the flask is split over 4 falcons and $\mathrm{MeOH}$ is added. After centrifugation, the formed pellets are redispersed in one falcon with toluene. The solution is precipitated a second time using ethanol. Again, the formed pellet is redispersed in toluene. At this step the CQDs are centrifuged in pure toluene to get rid of the lamellar phase. The solid phase is discarded. The stable phase is transferred in a weighted falcon and finally precipitated using methanol. The solid is dried under vacuum for $30 \mathrm{~min}$ (in glove box vacuum chamber). Finally, CQDs are redispersed with a $50 \mathrm{mg} \cdot \mathrm{mL}^{-1}$ concentration in toluene. 


\section{Material Characterization}

For infrared spectroscopy, we use a Fischer Nicolet iS50 in Attenuated Total Reflection (ATR). The spectra are averaged over 32 acquisitions and have a $4 \mathrm{~cm}^{-1}$ resolution.

X-ray diffraction is acquired using a Philips X-pert diffractometer operated under $40 \mathrm{kV}$ and $40 \mathrm{~mA}$. The X-ray beam is obtained from a Cu Ka line corresponding to a wavelength of $0.154 \mathrm{~nm}$. The diffraction pattern obtained from a thin film of HgTe CQDs is consistent with the zinc blende phase of the material, see Figure $\mathrm{S} 1$.

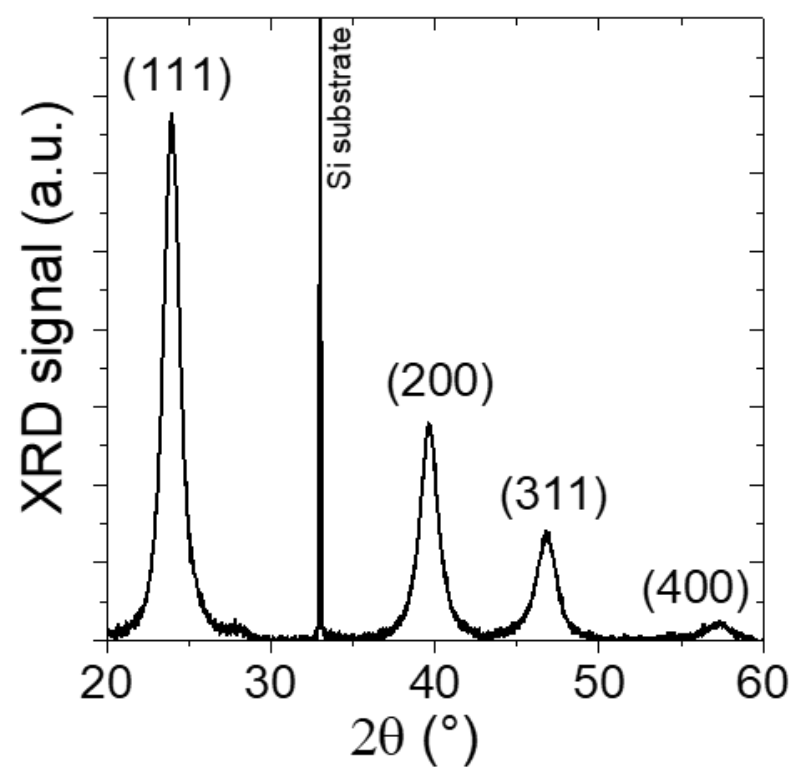

Figure S 1: X-ray diffraction pattern from a film made of $\mathrm{HgTe} C Q D$.

X-ray photoemission: Silicon wafer is rinsed with acetone, sonicated in acetone for 5 minutes. It is then rinsed again with acetone and isopropanol and dried with $\mathrm{N}_{2}$ gun. A $5 \mathrm{~nm}$ layer of $\mathrm{Cr}$ and an $80 \mathrm{~nm}$ layer of $\mathrm{Au}$ are deposited using thermal evaporation. A thin layer $(\approx 50 \mathrm{~nm})$ of CQDs is deposited on those substrates using the same procedure described for photoconductive device preparation. The samples are prepared as for transport measurements to avoid film charging.

The measurements are conducted on the Tempo beamline of Soleil synchrotron. The film is introduced in the preparation chamber, degassed for at least an hour and transferred into the analysis chamber. The measurements are conducted at $600 \mathrm{eV}$. This photon energy is precisely measured using the first and second order of $4 \mathrm{f}$ core level of Au using the formula: $h v_{\text {exp }}=K E_{2^{n d}}-$ $K E_{1}$ st.

The work function of the analyzer $\left(\mathrm{WF}_{\mathrm{A}}\right)$ is determined by measuring the kinetic energy of electrons at the Fermi level for the gold (set to $0 \mathrm{eV}$ ). $W F_{A}=h v_{\text {exp }}-K E_{\text {Fermi }}$

Analysis of the core level reveals the presence of $\mathrm{Hg}$ and Te contribution, see Figure S 2. C 1s and $S 2 p$ peaks are the results of EDT ligand. No contribution from phosphine (binding energy of $P 2 p$ state is $135 \mathrm{eV}$ ), possibly coming from Trioctylphosphine (Te precursor), is observed. 


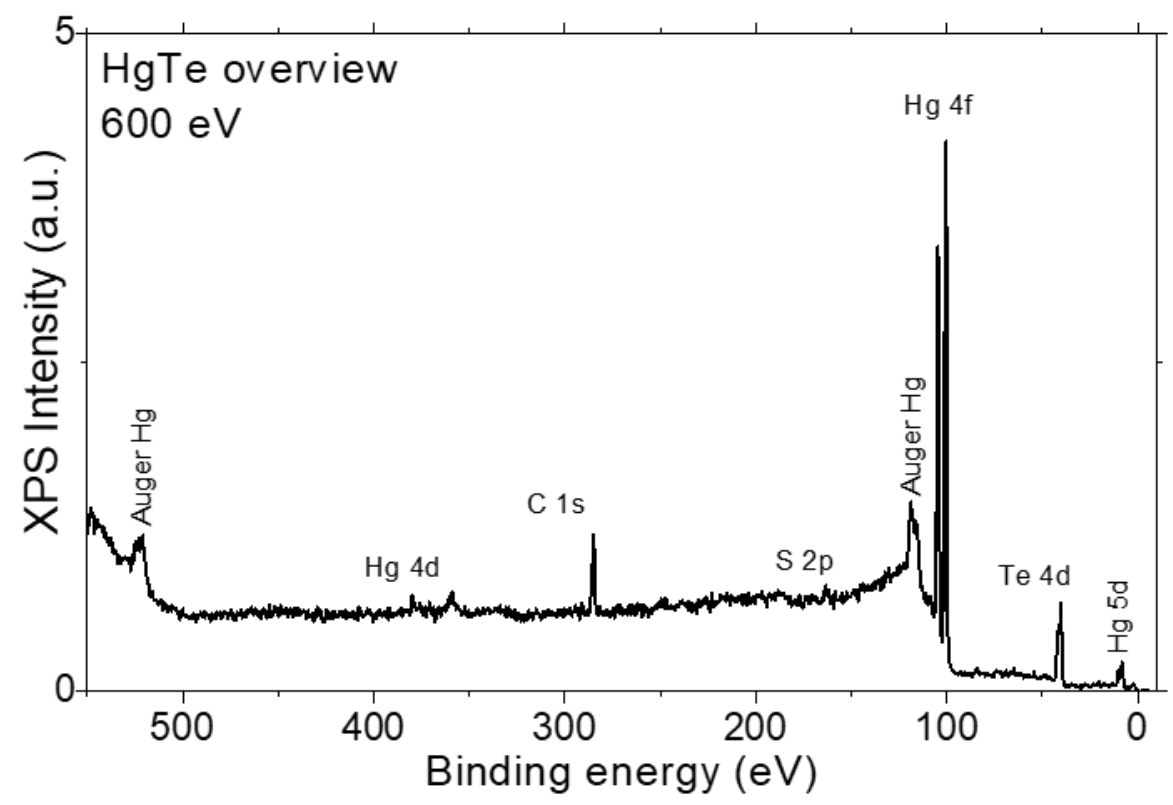

Figure S 2: XPS overview for a thin film of HgTe capped with EDT.

\section{TEM/STEM and EDX analysis}

TEM/STEM observations are made on a Titan Themis 200 microscope (FEl/ Thermo Fischer Scientific) equipped with a geometric aberration corrector on the probe. The microscope is also equipped with the "Super-X" systems for EDX analysis with a detection angle of 0.9 steradian. The observations are made at $200 \mathrm{kV}$ with a probe current of about $35 \mathrm{pA}$ and a half-angle of convergence of 17 mrad. HAADF-STEM images are acquired with a camera length of $110 \mathrm{~mm}$ (inner/outer collection angles were respectively 69 and 200 mrad). EDX probe is calibrated using bulk $\mathrm{HgCdTe}$ alloy with large $\mathrm{Hg}$ content grown from liquid phase epitaxy on $\mathrm{CdZnTe}$ substrate.

Figure $S 3$ and Figure $S 4$ respectively show high resolution imaging of the tripods and the EDX mapping of the sample. 


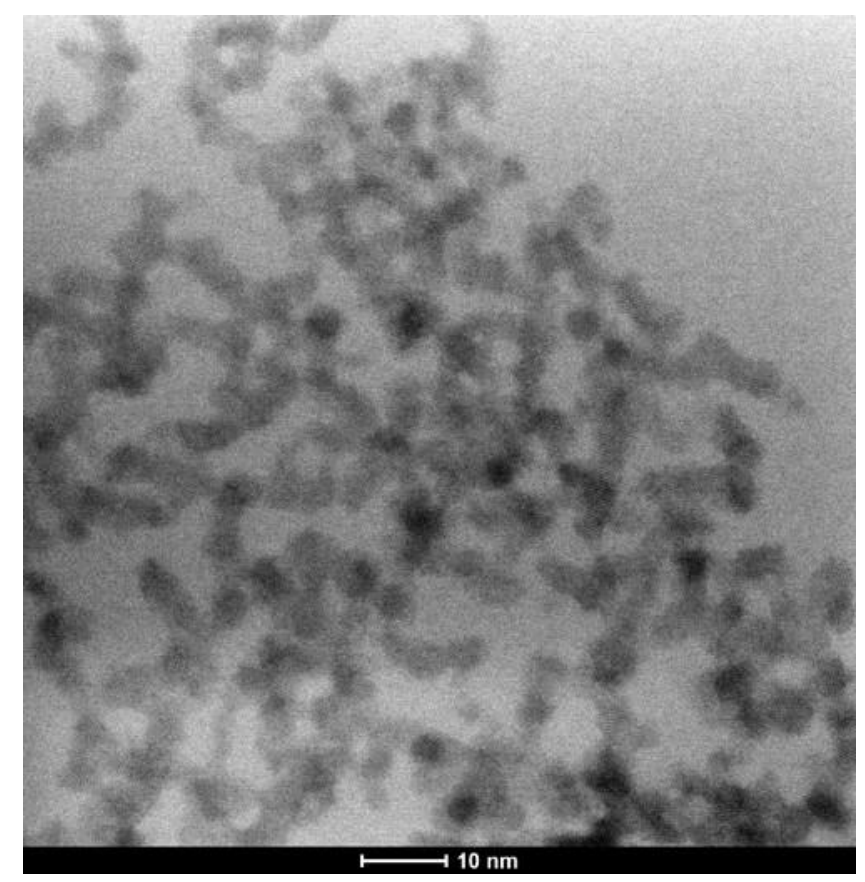

Figure S 3: High resolution TEM image of tripod shape HgTe CQDs.

EDX mapping of the tripods presents a clear correlation between the localization of the nanocrystals observed by STEM and the presence of $\mathrm{Hg}$ and $\mathrm{Te}$ atoms. On the other hand, oxygen mapping, does not present any correlation, thus we can exclude the formation of oxide on the HgTe tripod surface. This observation is consistent with XPS analysis where only evidence of $\mathrm{Hg}$ coupled to $\mathrm{S}$ (from ligands) and Te (from particle bulk) is observed. Quantitative EDX reveals that the nanocrystals are Te reach (Te atomic ratio is found to be $55 \%$ ). This result contrasts with previous measurements of EDX using SEM in which material have been found to be quasi stoichiometric. However, using SEM, the substrate is known to affect the measurement, which likely affect the obtained $\mathrm{Hg}: \mathrm{Te}$ ratio. EDX coupled to TEM, drastically reduces the impact of substrate. We nevertheless cannot fully exclude that ultra-high vacuum used for TEM operation is not evaporating $\mathrm{Hg}$ surface atoms. 


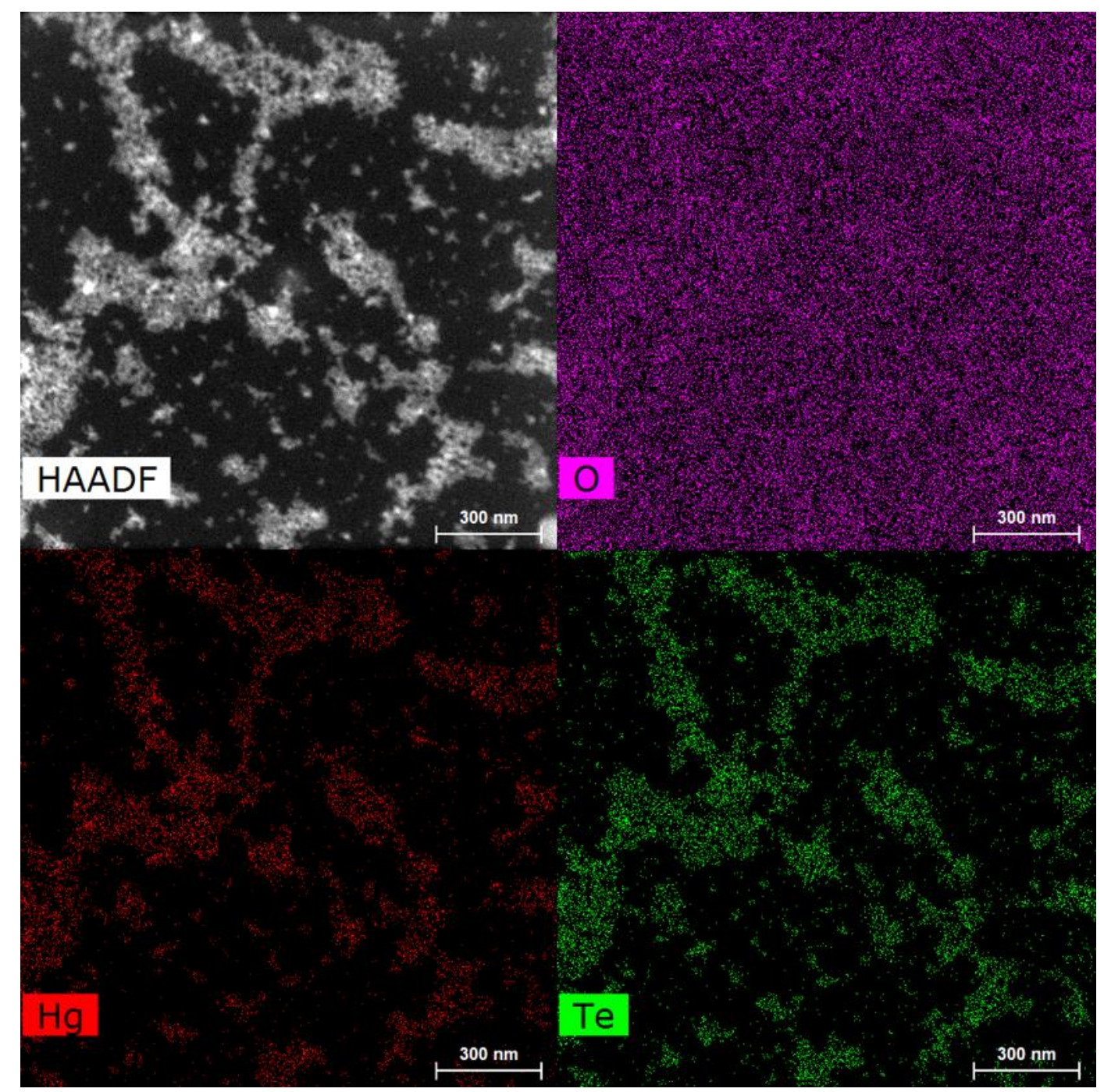

Figure S 4: STEM HAADF and EDX (from $\mathrm{Hg}, \mathrm{Te}$ and $\mathrm{O}$ atoms) mapping of tripod shape $\mathrm{HgTe}$ CQDs.

Electron tomography of tripod material: Electron tomography of a single CQD was performed using a Thermo Fisher Tecnai Osiris electron microscope operating at $200 \mathrm{kV}$. A tilt series of highangle annular dark-field transmission electron microscopy (HAADF-STEM) images were acquired from $-75^{\circ}$ to $69^{\circ}$, with a tilt increment of $3^{\circ}$. The projection images served as input for the $3 \mathrm{D}$ reconstruction performed through 20 iterations of the expectation maximization (EM) algorithm ${ }^{2}$ implemented in the ASTRA toolbox. ${ }^{3}$ The resulting 3D reconstruction was then segmented by defining a threshold value using Amira 5.4.0. The volume was extracted in a form of a 3D matrix used as input for the tight binding simulation.

In Figure S 5, we provide additional microscopy images and tomography reconstruted morphologies for $\mathrm{HgTe}$ CQD confirming their tripodic aspect. 


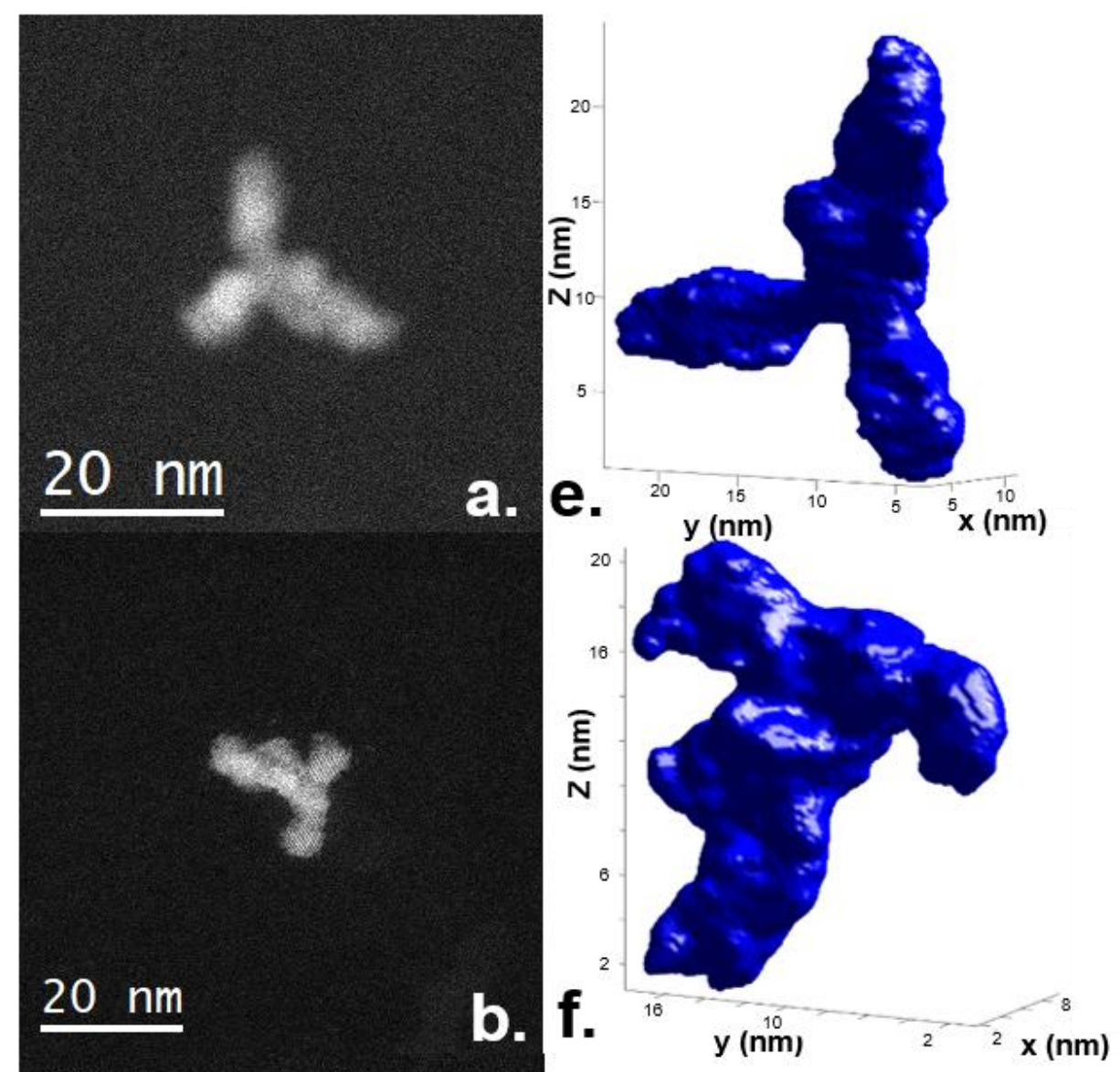

\section{$10 \mathrm{~nm}$}

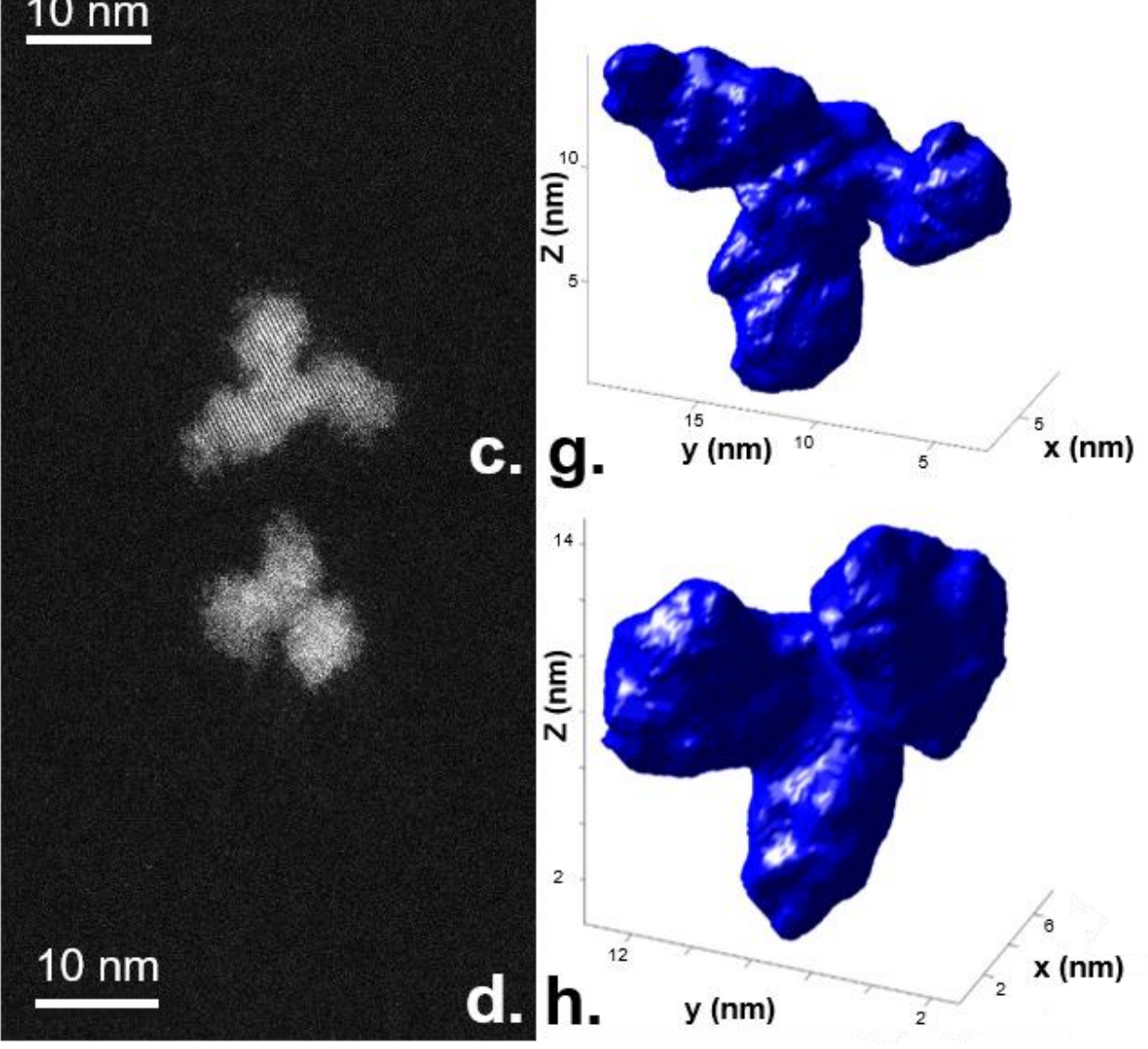

Figure S 5: a-d. Annular dark-field scanning transmission electron microscopy picture of a $\mathrm{HgTe}$ $C Q D$. e-h are tomography reconstructed morphologies of $\mathrm{HgTe} C Q D$ s observed respectively in a to $d$. 


\section{Electronic structure simulations}

Tight binding simulations: We used the tight binding model of Ref 4 to calculate the electronic structure of the HgTe CQDs. Each Hg or Te atom is described by a double set of $s p^{3} d^{5} s^{*}$ orbitals, one for each spin orientation. Surfaces are saturated by pseudo-hydrogen atoms characterized by a single s orbital. Tight-binding parameters, i.e., on-site energies, nearest-neighbor hopping matrix elements and spin-orbit coupling terms were determined to provide a very good description of the band structure of bulk HgTe (at $300 \mathrm{~K}$ ). For the CQD analyzed with electron tomography, we considered all atoms positioned on the zinc-blende lattice and included in the CQD volume $(\approx 12500$ atoms). For all QDs, we calculated 60 (1200) conduction (valence) states and we computed dipolar matrix elements between them as described in Ref 4.

The obtained spectra are then broadened by Gaussians so that the density of state is given by $\rho(E)=\frac{1}{\sqrt{2 \pi} \eta} e^{-\frac{\left(E-\left(E_{c, 0}-E_{v, 0}\right)\right)^{2}}{2 \eta^{2}}}$. The linewidth is continuously increased as described in ref 5 .

\section{Evaluation of the absorption spectrum}

This actual shape is then used as an input for atomistic tight-binding simulations of the electronic structure. The absorption spectrum is calculated using the Fermi Golden rule. ${ }^{4,6,7}$ We also simulated the spectrum for a spherical particle containing the same number of atoms ( $9.2 \mathrm{~nm}$ as diameter). In the latter case the tripod band-edge is clearly blue-shifted, compared to the sphere particle of 9.2 $\mathrm{nm}$, see Figure $S 6$. The spectrum associated to a sphere, composed by $1 / 3$ of the number of atoms, (6.4 $\mathrm{nm}$ as diameter) has also been evaluated. This volume corresponds to one arm of the tripod. In this case, the band-edge is strongly blue-shifted compared to the tripod, confirming that the tripod branches are electronically coupled. It is worth noticing that the band-edge energy appears to be smaller for the simulated data than the observed ones. It suggests that the selected particles observed in tomography are among the largest ones present in the solution or that a possible ripening of the CQDs had happened over time.

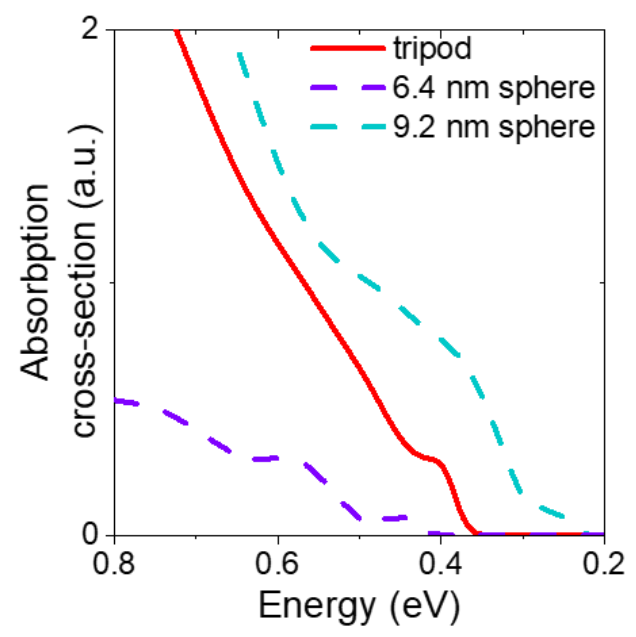

Figure S 6: Absorption spectra from tight binding simulations for $\mathrm{HgTe}$ tripod as revealed from tomography and for a sphere with the same number of atoms (12500 atoms, $9.2 \mathrm{~nm}$ as diameter) and for a sphere with a 1/3 of the atoms (6.4 $\mathrm{nm}$ as diameter) corresponding to the volume of one tripod arm. 


\section{Estimation of the wave function overlap:}

The calculation of the overlap between electron and hole wavefunctions in 3D is made using tight binding model described in Ref 4 . To estimate the wavefunction overlap, we first calculate the weight of the wavefunction on each atom according to these formulas:

$$
\begin{aligned}
c_{i} & \equiv \int_{\Omega_{i}}\left|\varphi_{e}(r)\right|^{2} d V \\
v_{i} & \equiv \int_{\Omega_{i}}\left|\varphi_{h}(r)\right|^{2} d V
\end{aligned}
$$

where $\Omega_{i}$ is the volume occupied by the atom $i$.

Secondly the overlap is defined as:

$$
S=\frac{2 \sum_{i} c_{i} v_{i}}{\sum_{i}\left|c_{i}\right|^{2}+\sum_{i}\left|v_{i}\right|^{2}}
$$

Using this definition, $S$ is equal to one when the two wavefunctions are identical and goes to zero when those two do not overlap.

Table $S 1$ Electron hole wave function associated with the ground state overlap for various shapes of $\mathrm{HgTe} C Q D$.

\begin{tabular}{lc}
\hline Particle & $\begin{array}{c}\text { Wave function } \\
\text { overlap }\end{array}$ \\
\hline Tripod 1 see figure 1d and Figure S 7 & 0.40088 \\
\hline Tripod 2 see Figure S 8 and Figure S 9 & 0.42388 \\
\hline Sphere 9.2 nm, see Figure S 10 & 0.39460 \\
\hline
\end{tabular}




\section{Planar tripod}

While Figures 1d-e present a 3D view of the wave function, Figure S 7 and Figure S 9 provide the planar projection of the wavefunction for two tripods. We clearly observe the anisotropic shape of the wavefunction with one direction $(\mathrm{y}, \mathrm{z})$ being much larger $(13 \mathrm{~nm})$ than the other $(5-10 \mathrm{~nm})$. This projection also confirmed that the hole wavefunction is more confined than the electron one.
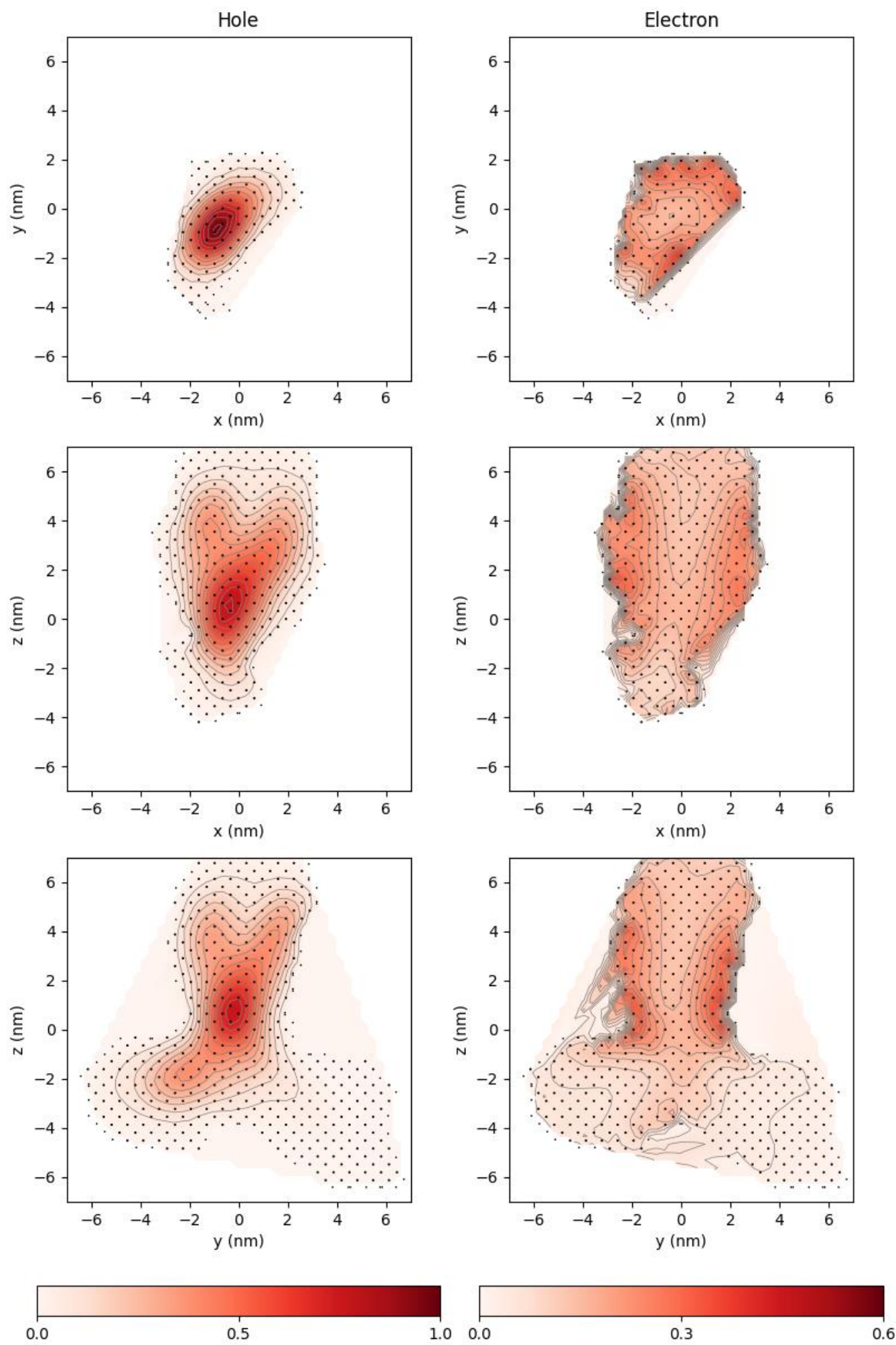

Figure S 7: Left (resp. right) projection of the hole (resp. the electron) wavefunction along the three directions for the HgTe tripod which shape is depicted in Figure 1. 


\section{Non planar tripod}

As electron microscopy may select a non-representative particle, we have conducted tomography on several HgTe CQDs. The tripodic shape seems consistent over the sample. However, the arms can also be out of plane, see Figure $S 8$. The planar projections of the wave function given in Figure $\mathrm{S} 8 \mathrm{~b}$ and $\mathrm{c}$ are given in Figure S 9. Main conclusion (non-isotropy of the shape and stronger hole confinement) remains unchanged for this second particle.

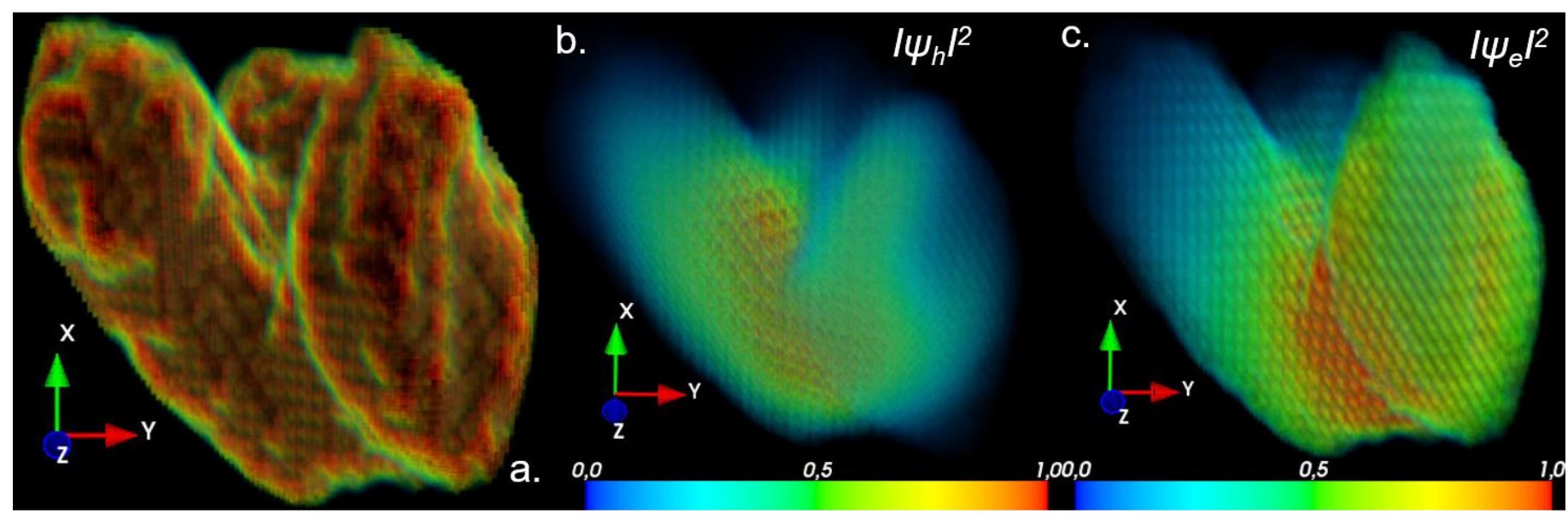

Figure S 8: a. Reconstruted shape from a second (non planar tripod) HgTe CQD as revealed from electron tomography. b. (resp. c.) Square modulus of the 3D hole (resp. electron) wavefunction for an $\mathrm{HgTe}$ tripod as revelaed from tomography. 

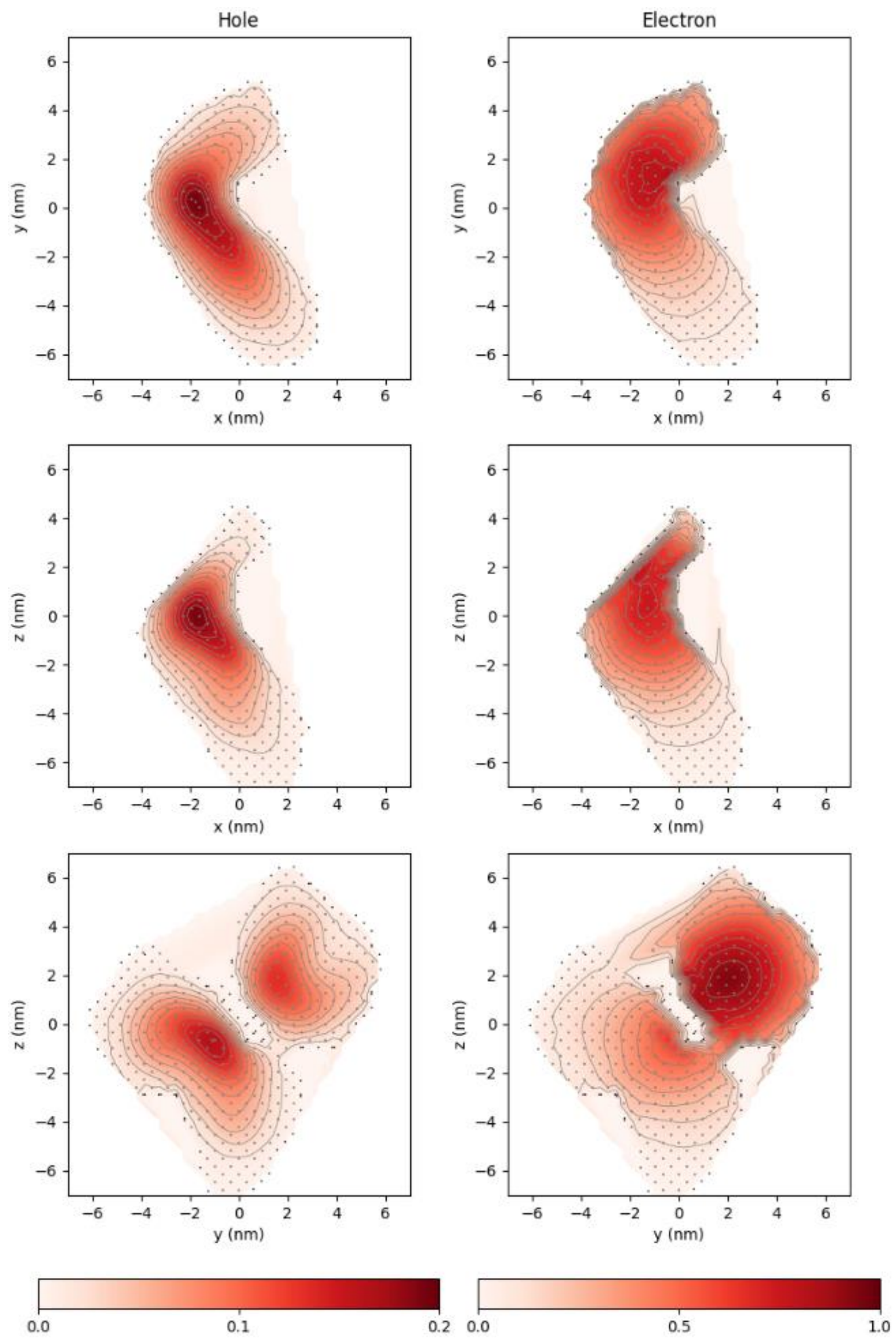

Figure S 9: Left (resp. right) projection of the hole (resp. the electron) wavefunction along the three directions for the HgTe tripods which shape is depicted in Figure $S 8$. 


\section{Sphere shape particle}

For sake of comparison, similar tight binding simulations has been conducted with a $\mathrm{HgTe}$ sphere, which size includes the same number of atoms as the tripod from Figure 1. The obtained wave functions have obviously a symmetry. On the other hand, as for the tripod, the hole, due to its higher mass, presents a density of presence mostly localized in the center of the particle.
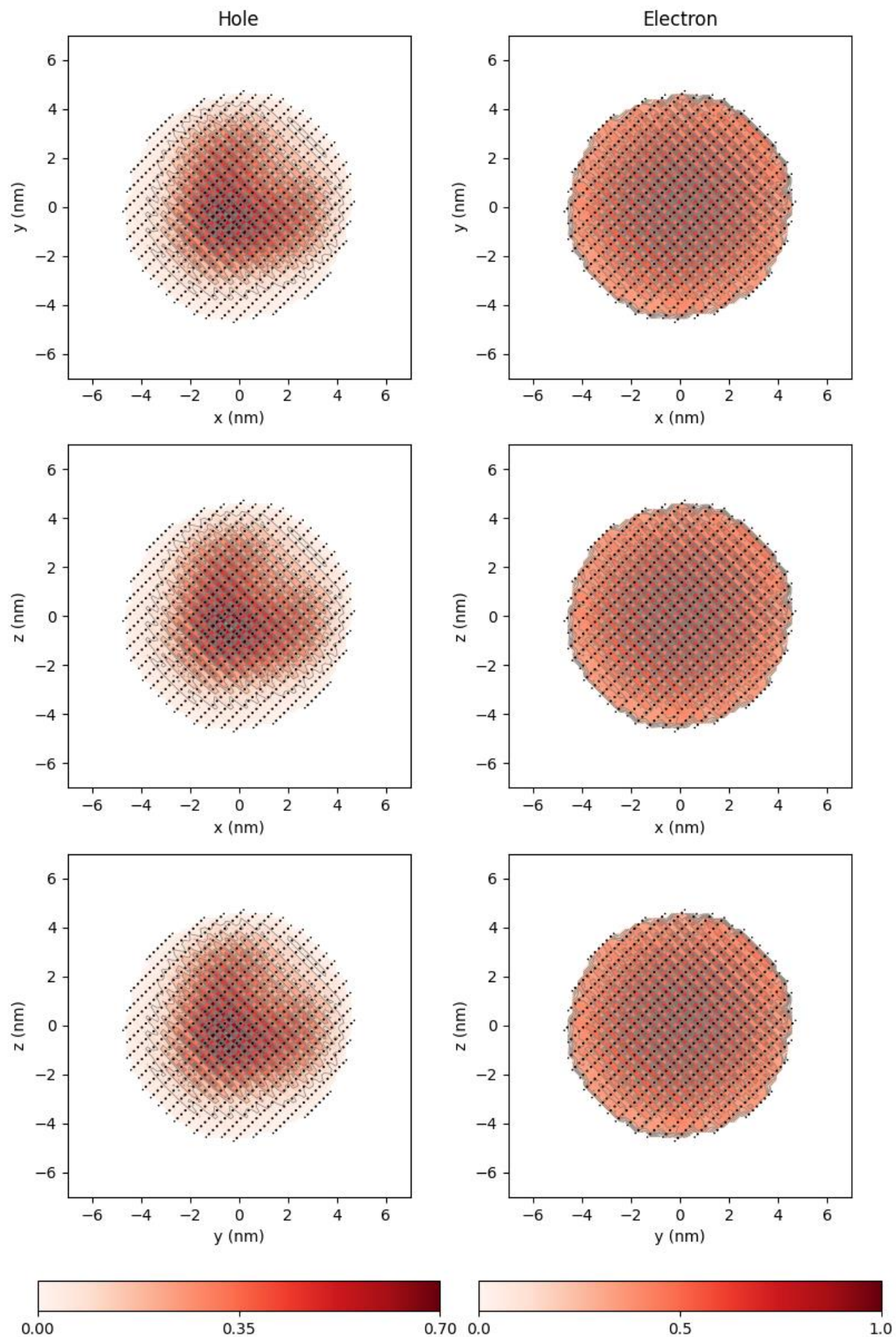

Figure S 10: Left (resp. right) projection of the hole (resp. the electron) wave function along the three direction for the HgTe sphere, which number of atoms (12500) is the same as the one included in the tripod. 


\section{Dual-gate device fabrication}

Dual gate electrodes fabrication: All electrodes are patterned using a standard photolithography technique. First, gate electrodes (gap distance: $3 \mu \mathrm{m}$ ) and source/drain contact pads are patterned onto a $\mathrm{Si} / \mathrm{SiO}_{2}$ substrate $\left(400 \mathrm{~nm}\right.$ thick $\mathrm{SiO}_{2}$ ), followed by consecutive metal deposition using a thermal evaporator given as follow: $5 \mathrm{~nm}$-thick $\mathrm{Cr}, 40 \mathrm{~nm}$-thick $\mathrm{Au}$, and $5 \mathrm{~nm}$ Al. This last layer is there to promote the adhesion of the subsequent alumina layer. After liftoff, we deposit $40 \mathrm{~nm}$ of $\mathrm{Al}_{2} \mathrm{O}_{3}$ onto devices by $\mathrm{ALD}$, which serves as a gate dielectric. Next, $\mathrm{Al}_{2} \mathrm{O}_{3}$ areas onto contact pads of gates and source/drain are etched by $\mathrm{H}_{3} \mathrm{PO}_{4}(85 \%)$ solution at $50{ }^{\circ} \mathrm{C}$ for 6 min, to improve contact properties. Subsequently, source/drain electrodes connected to pads are patterned, followed by metal deposition (5 $\mathrm{nm}$ thick $\mathrm{Cr}$ and $100 \mathrm{~nm}$ thick $\mathrm{Au}$ ).

Figure S 11 depicts the different steps of fabrication of the dual-gate transistor, while Figure S 12 provides the geometrical factor of the device.
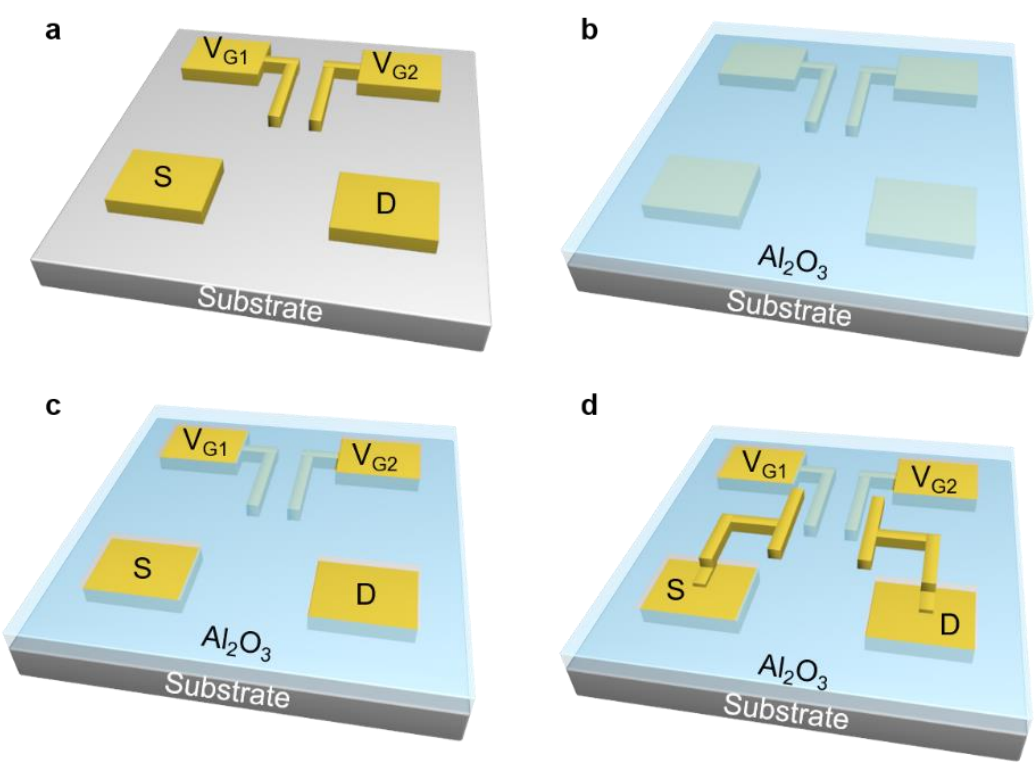

Figure S 11: Main steps relative to the fabrication of the dual gate device. a. During step 1, the contact pads and the two gates are defined. b. During step 2, the whole device is covered by an $\mathrm{Al}_{2} \mathrm{O}_{3}$ insulating layer, later used as high-k material. $\mathrm{c}$. The alumina covering the contact pads is etched using $\mathrm{H}_{3} \mathrm{PO}_{4}$. d. Finally, step 4 is used to defined drain and source contacts.

HgTe film deposition onto dual gate devices: A solution of $\mathrm{HgTe} 4 \mathrm{k}$ CQDs (cut-off at $4000 \mathrm{~cm}^{-1}$, $25 \mathrm{mg} \cdot \mathrm{mL}^{-1}$ in toluene) is spin-coated in air onto electrodes at $2000 \mathrm{rpm}$ for $1 \mathrm{~min}$. To perform the ligand exchange, the spin-coated device is dipped in asolution of $1 \% 1,2$-ethanedithiol (EDT) in acetonitrile for $30 \mathrm{sec}$, and then rinsed in fresh acetonitrile. This step is repeated several times to reach a final thickness between 50 and $200 \mathrm{~nm}$. 


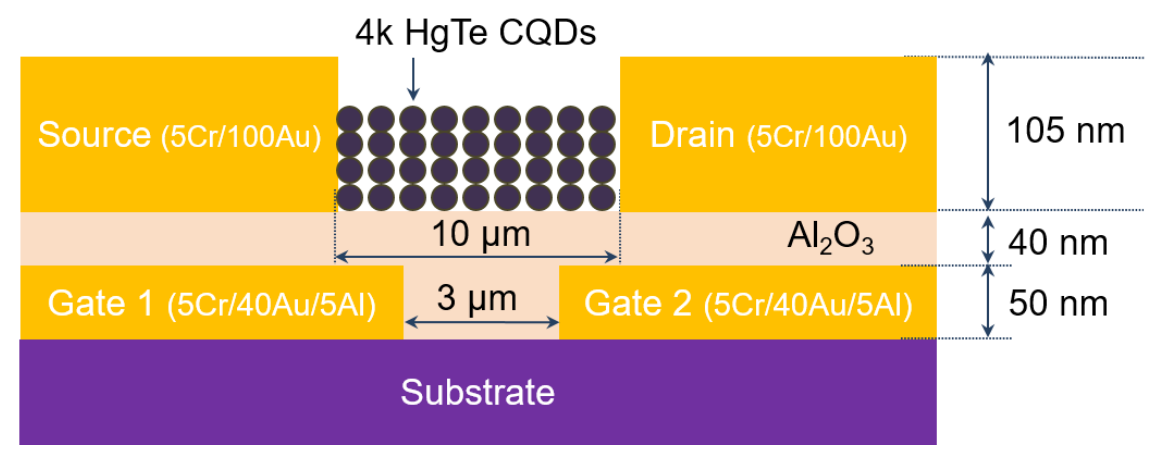

Figure S 12 Sketch of the dual gate device crossection.

In the dual gate device, the carrier density $\rho$ induced by the gate is given by $\rho=\frac{\mathrm{C}_{\Sigma} V_{G}}{t}$ with $\mathrm{C}_{\Sigma}$ the gate sheet capacitance, $V_{G}$ the applied gate bias, and $t$ the film thickness $(100 \mathrm{~nm}) . \mathrm{C}_{\Sigma}$ is given by $\mathrm{C}_{\Sigma}=\frac{\varepsilon_{A l 2 O 3} \varepsilon_{0}}{t_{\text {gate }}}$ with $\varepsilon_{A l 2 O 3}=7.5$ the alumina dielectric constant, $t_{\text {gate }}$ the alumina gate thickness, and $\varepsilon_{0}$ the vacuum permittivity. Under $4 \mathrm{~V}$ of gate bias, the induced carrier density corresponds to $4 \times 10^{19}$ carriers. $\mathrm{cm}^{-3}$, which corresponds to several carriers per CQD.

\section{Hysteresis and mobility for dual gate device}

Hysteresis is observed in the FET transfer curve. The latter is associated to the filling of the traps on the surface of the oxide. We can estimate their density $\mathrm{n}=\mathrm{C}_{\Sigma} \cdot \Delta V_{\text {hys }}$ with $\Delta V_{\text {hys }}$ the hysteresis bias $\approx 5 \mathrm{~V}$, leading to $\mathrm{n} \approx 5 \times 10^{12} \mathrm{~cm}^{-2}$. The mobility of the carrier has been estimated at $250 \mathrm{~K}$ to be in the $10^{-3} \mathrm{~cm}^{2} . \mathrm{V}^{-1} \mathrm{~s}^{-1}$ range, consistent with previous measurements for HgTe CQD capped with EDT. ${ }^{8}$

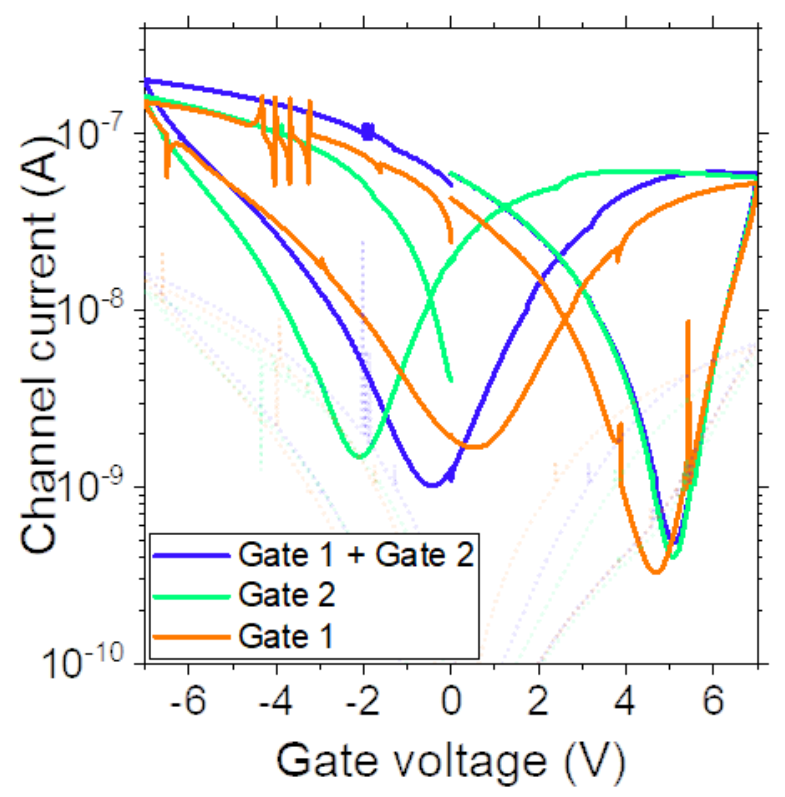

Figure S 13: Transfer curves (drain and gate currents as a function of the applied gate bias while the drain source bias is set to $1 \mathrm{~V}$ ) while gate 1 , gate 2 (with the other gate is floating), or gate $1+$ gate 2 are tuned. 


\section{Dual-gate device operation}

Electrical characterization: The sample is mounted on the cold finger of a closed cycle He cryostat. Unless specified, the measurements have been conducted at $250 \mathrm{~K}$ under secondary vacuum. Both gates are connected to the two channels of a Keithley 2634B, while the channel is connected to another Keithley 2634B used to apply a channel voltage and measure the resulting current. Measurements under illumination are obtained while the sample is illuminated by a $1.55 \mu \mathrm{m}$ laser diode.

Figure S 14 discuss the effect of the film thickness on the FET (Figure S 14a) and diode (Figure S $14 \mathrm{~b})$ performances. From Figure $S 14 \mathrm{a}$ one can see that current modulation is mostly preserved while the channel thickness is increased from 50 to $200 \mathrm{~nm}$. Figure $S 14 \mathrm{~b}$ provides the Voc map for the dual gate FET coupled to a thin $(50 \mathrm{~nm})$ film.
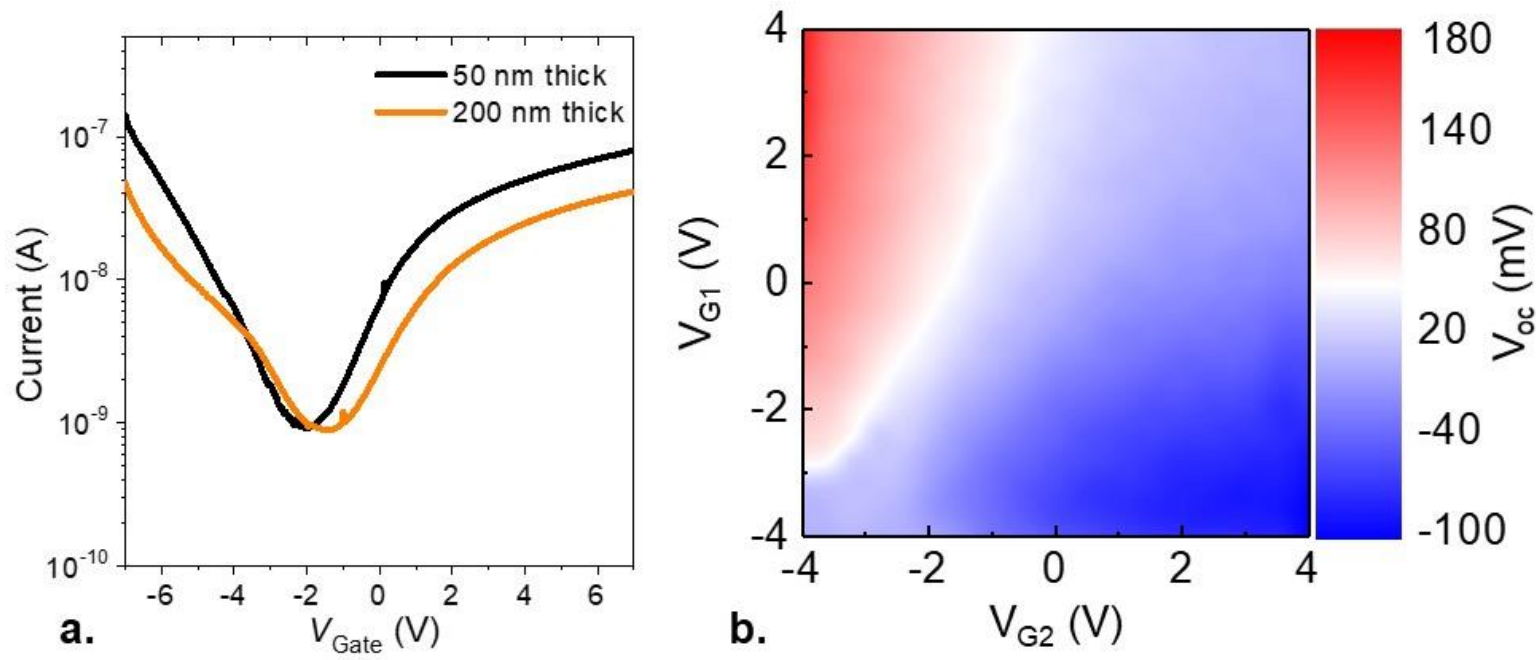

Figure S 14: a. Tranfer curves associated with a $50 \mathrm{~nm}$ and $200 \mathrm{~nm}$ thick film of $\mathrm{HgTe}$ CQD film. $b$. Open circuit voltage map as a function of applied gate 1 and gate 2 biases for a thin (50 nm) $\mathrm{HgTe}$ CQD-based channel.
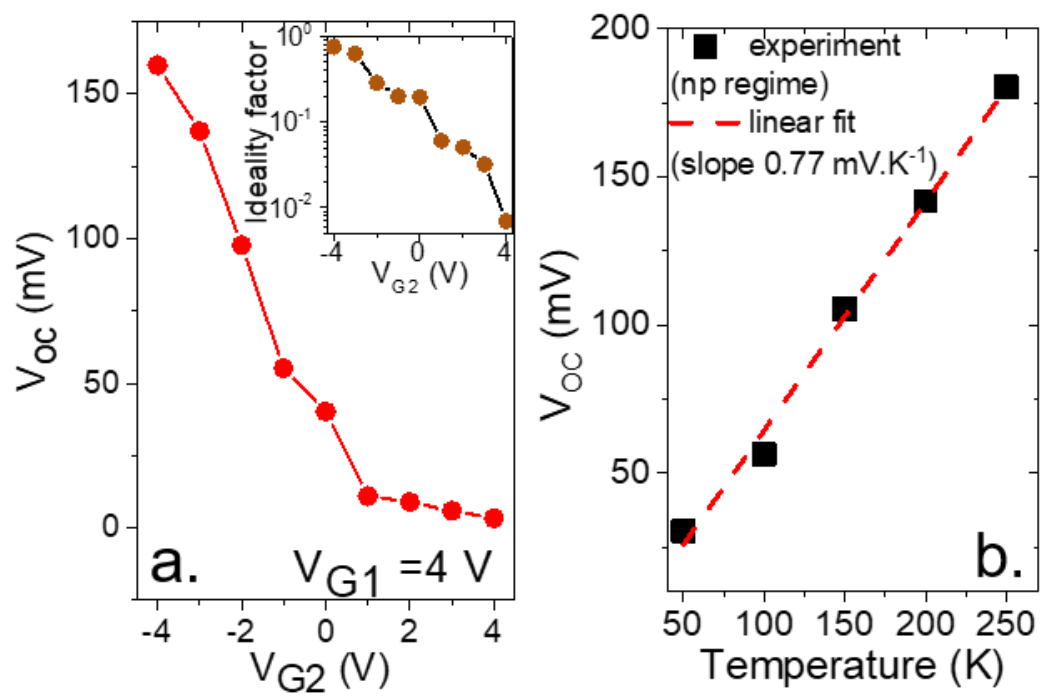

Figure S 15: a. Open circuit voltage under an irradiance of $1 \mathrm{~W} . \mathrm{cm}^{-2}$ at $1.55 \mu \mathrm{m}$ as a function of $V_{G 2}$ while $V_{G 1}$ is set at $+4 \mathrm{~V}$. The inset is the diode ideality factor as a function of $V_{G 2}$. $b$. Open circuit voltage as a function of the temperature while the device is operated in the $n$-p regime. 
As the gate 2 bias is made opposite to gate 1 bias, the dual gate FET device switches from phototransistor regime to a diode behavior, see Figure $S 15 \mathrm{a}$. Figure $S 15 \mathrm{~b}$ shows temperature dependance of the open circuit voltage of the dual-gate FET. It is noteworthy that the slope of the graph $V_{o c}$ vs power, that relates to the ideality factor $\left(n_{i d}\right)$, is much higher for the diode $\left(n_{\text {id }}=1.5\right)$ than for the dual gate device ( $\mathrm{n}_{\mathrm{i}}=0.8$ maximum according to inset of Figure $\mathbf{S} 15 \mathrm{a}$ ). Such low ideality factor (i.e. below 1 ) is typically associated with non-selective contact diode, ${ }^{9}$ which may have been anticipated since both drain and source are made of gold.

\section{Dual-gate device performances for photodetection}

\section{Effect of bias on responsivity}

Figure S 16 provides responsivity map for a $200 \mathrm{~nm}$ thick film made of tripodic $\mathrm{HgTe}$ CQDs and operated under 3 different channel biases. While $0 \mathrm{~V}$ operation is interesting to minimize the dark current, we clearly observe that the application of a small drain source bias leads to an increase of photoresponse by a factor 5 to 10 .
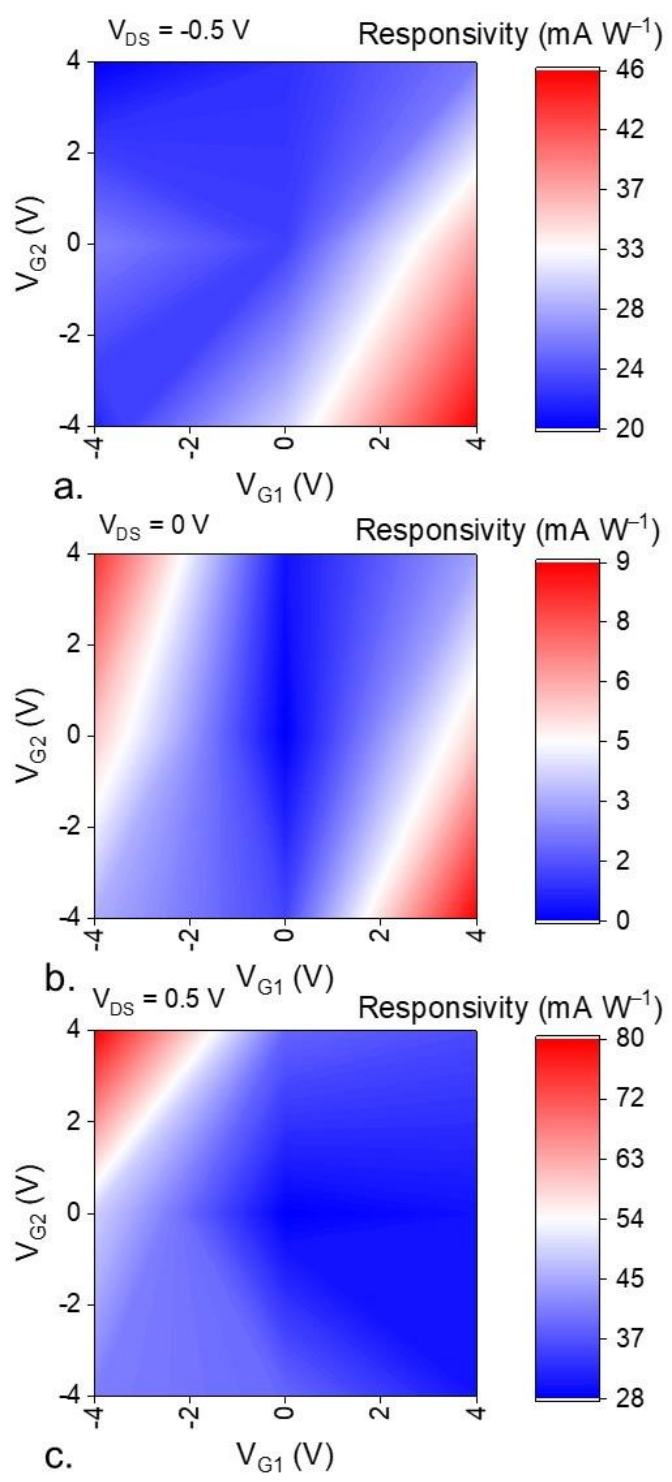
Figure S 16: Open circuit voltage map as a function of applied gate 1 and gate 2 biases for a 200 $\mathrm{nm}$ thick film HgTe CQD-based channel operated under $-0.5 \mathrm{~V}$ (a.), $0 \mathrm{~V}$ (b.) and $+0.5 \mathrm{~V}$ (c.) drain source bias.

Figure $S 17$ summarizes the effect of the operation mode (diode vs transistor) and applied drain source bias $(0$ or $-0.5 \mathrm{~V})$ on the responsivity. At $0 \mathrm{~V}$ the benefit of the diode, is very striking with an increase of the response by a factor 50 . Under an applied bias, the difference of responsivity between the two modes becomes weaker, and the benefit of low dark current for the diode is also mostly lost. However, the absolute value of the response is clearly increased and achieved values are above $>10 \mathrm{~mA} \cdot \mathrm{W}^{-1}$ at moderate power.

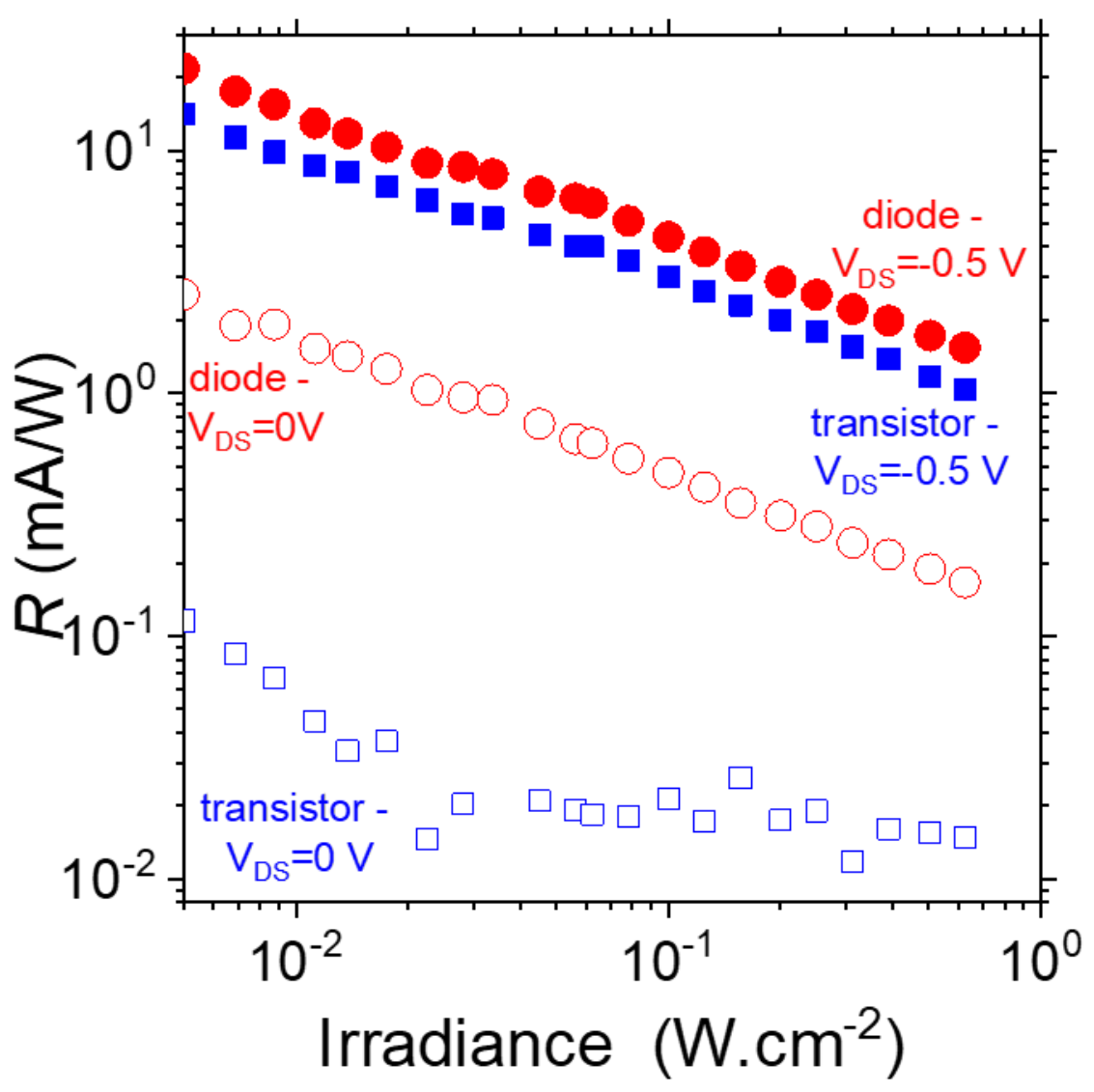

Figure S 17: Effect of applied drain source bias and operation mode (transistor vs diode) on the responsivity of the device as a function of the light irradiance. 


\section{Detectivity estimation}

To determine the signal to noise ratioand the specific detectivity, noise has to be estimated. However, the current setup clearly limits the noise estimation. The presence of the two gates brings strong $50 \mathrm{~Hz}$ noise and many of its harmonics, see Figure $S$ 18. As a result, the obtained noise spectral density is not a white noise, presents neither a $1 / f$ dependence, nor a bias dependence. This is a clear difficulty resulting from the addition of electrodes. They can indeed bring more control and enable a p-n junction formation but also bring noise which makes the measurement set-up limited. As a result, this noise measurement can be seen as an upper limit of the noise in the sample.

To obtain a lower limit of the noise value we can assume that noise will be higher that the shot noise. The latter can be estimated from an analytical expression: Sishot $=(2 \mathrm{el})^{1 / 2}$, where $e$ is the proton charge and / the flowing current.

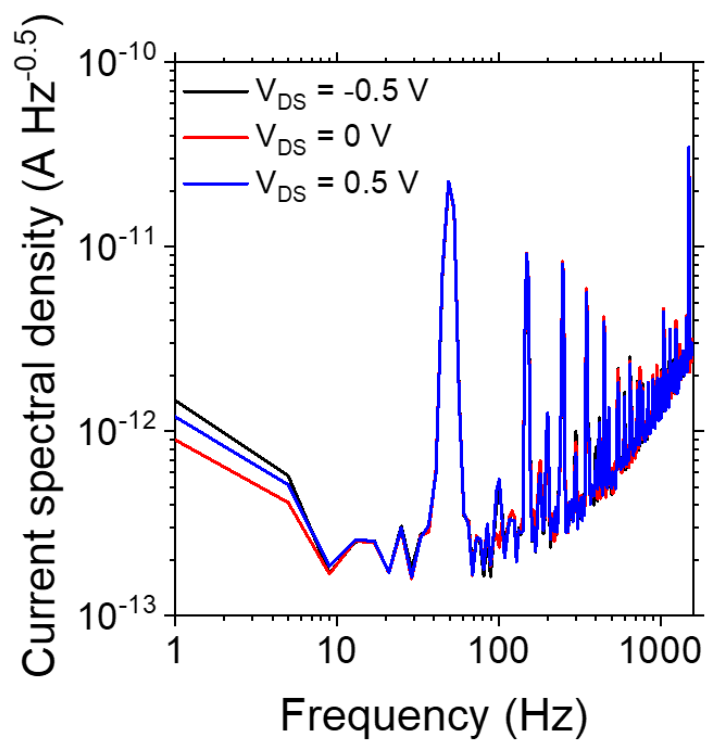

Figure S 18: Current spectral density for a $200 \mathrm{~nm}$ thick film HgTe CQD-based channel operated under 3 different biases.

Table S 2 Responsivity and specific detectivity estimation for a $200 \mathrm{~nm}$ thick film HgTe CQD-based channel at $250 \mathrm{~K}$.

\begin{tabular}{|c|c|c|c|}
\hline & $\begin{array}{c}\text { Responsivity } \\
\left(\mathrm{mA} \cdot \mathrm{W}^{-1}\right)\end{array}$ & $\begin{array}{c}\text { Detectivity estimation } \\
\text { from Figure S } 18 \\
\text { (Jones) }\end{array}$ & $\begin{array}{c}\text { Detectivity estimation } \\
\text { from shot noise } \\
\text { (Jones) }\end{array}$ \\
\hline $\begin{array}{c}V_{G 1}=4 \mathrm{~V}, V_{G 2}=-4 \mathrm{~V} ; \\
V_{D S}=0 \mathrm{~V}\end{array}$ & 9 & $9 \times 10^{7}$ & $1.5 \times 10^{10}$ \\
\hline $\begin{array}{c}\mathrm{V}_{\mathrm{G} 1}=4 \mathrm{~V}, \mathrm{~V}_{\mathrm{G} 2}=-4 \mathrm{~V} ; \\
\mathrm{V}_{\mathrm{DS}}=0.5 \mathrm{~V}\end{array}$ & 80 & $4.6 \times 10^{8}$ & $3.3 \times 10^{10}$ \\
\hline
\end{tabular}




\section{Comparison of dual gate device for SWIR sensing with state-of-the-art devices}

Table S 3 State of the art for SWIR and extended SWIR operating HgTe nanocrystal-based detector. $P C, P T$ and $P D$ stand respectively for photoconductor, phototransistor and photodiode.

\begin{tabular}{|c|c|c|c|c|c|c|c|}
\hline $\begin{array}{l}\text { Cut-off } \\
\text { Wavele } \\
\text { ngth } \\
(\mu \mathrm{m})\end{array}$ & $\begin{array}{l}\text { Operating } \\
\text { mode }\end{array}$ & $\begin{array}{c}\text { Respons } \\
\text { ivity } \\
\left(A \cdot W^{-1}\right)\end{array}$ & $\begin{array}{c}\text { Response } \\
\text { time }\end{array}$ & Detectivity (jones) & $\begin{array}{l}\text { Operatin } \\
\mathbf{g} \\
\text { temperat } \\
\text { ure } \\
\text { (K) }\end{array}$ & Specific feature & Reference \\
\hline 2.5 & PC & 0.1 & $10 \mu \mathrm{s}$ & $3.5 \times 10^{10}$ & 230 & $\begin{array}{c}\mathrm{As}_{2} \mathrm{~S}_{3} \text { surface } \\
\text { chemistry }\end{array}$ & 8 \\
\hline 2.5 & $\mathrm{PC}$ & 1000 & $20 \mu \mathrm{s}$ & $2 \times 10^{12}$ & 200 & nanotrench & 10 \\
\hline 2.5 & PC & 150 & $1.5 \mathrm{~ms}$ & $6 \times 10^{8}$ & 80 & $\begin{array}{l}\text { HgTe decorated } \\
\text { graphene channel }\end{array}$ & 11 \\
\hline 2.5 & PT & $6.5 \times 10^{-3}$ & $10 \mu \mathrm{s}$ & $10^{9}$ & 220 & Graphene electrode & 12 \\
\hline 2.5 & PT & $2.0 \times 10^{-3}$ & $14 \mu \mathrm{s}$ & $10^{12}$ & 30 & STO gate+resonator & 13 \\
\hline 2 & PT & $<0.5$ & $\approx 10 \mu \mathrm{s}$ & $3 \times 10^{10}$ & 300 & $\mathrm{SiO}_{2}$ back gate & 14 \\
\hline 2.4 & PT & 1 & $1.5 \mu \mathrm{s}$ & $10^{10}$ & 300 & $\begin{array}{c}\text { Hybrid Polymer: } \\
\text { HgTe }\end{array}$ & 15 \\
\hline 2.5 & PD & $2.5 \times 10^{-3}$ & $370 \mathrm{~ns}$ & $3 \times 10^{9}$ & 300 & HgTe ink & 16 \\
\hline 2.5 & PD & 0.25 & $\begin{array}{l}12 \text { ns rise } \\
\text { time } \\
260 \text { decay } \\
\text { time }\end{array}$ & $\begin{array}{c}3 \times 10^{10} \\
\text { (without cavity) } \\
7.5 \times 10^{10 \text { (with cavity) }}\end{array}$ & 300 & Flexible substrate & 17 \\
\hline 2.2 & PD & 1 & $\begin{array}{c}450 \text { ns (rise) } \\
1.4 \mu \mathrm{s} \\
\text { (decay) }\end{array}$ & $6 \times 10^{10}$ & 300 & $\mathrm{HgCl}_{2}$ treatment & 18 \\
\hline 1.8 & PD & 0.13 & $110 \mathrm{~ns}$ & $2 \times 10^{10}$ & 300 & With resonator & 19 \\
\hline 2.5 & PD & 0.08 & $10 \mu \mathrm{s}$ & $\begin{array}{l}>10^{8} \\
<10^{10}\end{array}$ & 250 & Dual gate & This work \\
\hline
\end{tabular}

Table S 3 compares the dual gate device with state-of-the-art device used for SWIR sensing and based on $\mathrm{HgTe}$ nanocrystals. The value of the responsivity (a few $\mathrm{mA}^{\left.-\mathrm{W}^{-1}\right)}$ is actually typical from what can be obtained from an HgTe CQDs thin film in the hopping regime and in absence of any light trapping. It nevertheless remains lower that what can be achieved using photodiode geometry (100 to $1000 \mathrm{~mA}^{-W^{-1}}$ range) or using light resonator (with R typically above $500 \mathrm{~mA} . \mathrm{W}^{-1}$ ). Light absorption is clearly a limitation of the current dual gate geometry. Operation in the diode regime enable to achieve detectivity in the $10^{8}-10^{10}$ Jones range, which stay below best devices due to the low responsivity, but comparable with other low-cost technology. 


\section{Comparison HgTe CQD-based vertical geometry photodiode}

We have compared the open circuit voltage obtained from our planar dual gate FET with the one from the best performing $\mathrm{HgTe}$ CQD-based photodiode structure. The latter relies on a ITO/HgTe/Ag 2 Te/gold stack. ${ }^{20,21}$

$\mathrm{Ag}_{2} \mathrm{Te}$ CQD synthesis: In a $25 \mathrm{ml}$ three neck flask, $34 \mathrm{mg} \mathrm{AgNO}_{3}(0.2 \mathrm{mM}), 5 \mathrm{~mL}$ OLA and $0.5 \mathrm{~mL}$ $\mathrm{OA}$ are degassed at $70{ }^{\circ} \mathrm{C}$ under vacuum until the $\mathrm{AgNO}_{3}$ is completely dissolved and the solution becomes clear. Under nitrogen, $0.5 \mathrm{~mL}$ TOP is injected into the solution. Then temperature is raised to $160{ }^{\circ} \mathrm{C}$. At $160^{\circ} \mathrm{C}$ the solution becomes orange. Then $0.1 \mathrm{~mL}$ TOP:Te $(1 \mathrm{M})$ are injected into the solution and the reaction is quenched after $10 \mathrm{~min}$ with a water bath. The CQDs are precipitated with methanol and redispersed in chlorobenzene. At this step, $500 \mu \mathrm{L}$ of DDT are added. The washing step is repeated one more time and finally the CQDs are redispersed in a hexane:octane (9:1) solution.

$\mathrm{HgTe}$ CQDs synthesis with band-edge at $6000 \mathrm{~cm}^{-1}$ : In a $50 \mathrm{~mL}$ three-neck flask, $540 \mathrm{mg}$ of $\mathrm{HgCl}_{2}$ and $50 \mathrm{~mL}$ of oleylamine are degassed under vacuum at $110^{\circ} \mathrm{C}$. At this stage, the solution was yellow and clear. Meanwhile, $2 \mathrm{~mL}$ of TOP:Te $(1 \mathrm{M})$ are extracted from the glove box and mixed with $8 \mathrm{~mL}$ of oleylamine. The atmosphere is switched to $\mathrm{N}_{2}$ and the temperature is set at $57^{\circ} \mathrm{C}$. The pre-heated TOP:Te solution is quickly injected and the solution turned dark after $1 \mathrm{~min}$. After $3 \mathrm{~min}$, $10 \mathrm{~mL}$ of a mixture of $20 \%$ DDT in toluene is injected and a water bath is used to decrease quickly the temperature. The content of the flask is spilt in 4 falcons and $\mathrm{MeOH}$ was added. After centrifugation, the formed pellets are redispersed in one falcon with $10 \mathrm{~mL}$ of toluene. The solution is precipitated a second time with absolute $\mathrm{EtOH}$. Again, the formed pellet is redispersed in $8 \mathrm{~mL}$ of toluene. At this step, the CQDs are centrifuged in pure toluene to remove the lamellar phase. The solid phase is discarded and the supernatant is filtrated.

HgTe ink : $1 \mathrm{~mL}$ of $\mathrm{HgTe}$ CQD solution in toluene is mixed with $1 \mathrm{~mL}$ of exchange solution as previously reported. ${ }^{22} 5 \mathrm{~mL}$ of hexane is added to the solution and mixed with vortex and sonication. After a few seconds, two phases are clearly visible and the hexane one (top one) is removed. The solution is cleaned with hexane one more time. At this step, $250 \mu \mathrm{L}$ of DMF is added to avoid precipitation. The solution is cleaned again with hexane. Then, the solution is precipitated by adding ethanol and centrifuged at $6000 \mathrm{rpm}$ for $4 \mathrm{~min}$. Supernatant is discarded and the CQDs are redispersed in $130 \mu \mathrm{L}$ of DMF.

Diode fabrication : The procedure is inspired from ref 20,21 . For the device fabrication, $70 \mathrm{~nm}$ FTO $\left(\rho_{s} \approx 70-90 \Omega / s q\right)$ on glass substrate from Solems are used. To define the device area, FTO electrodes are patterned with optical UV lithography. The HgTe CQD ink (band edge at $6000 \mathrm{~cm}^{-1}$ ) is deposited onto the patterned FTO substrate via spin-coating. The thickness of the film is tuned with spin-coating speed and ink concentration in DMF solvent. On top of $\mathrm{HgTe}$ ink film $\mathrm{Ag}_{2} \mathrm{Te} \mathrm{CQD}$ layer is spin-coated at $2000 \mathrm{rpm}$ followed by $\mathrm{HgCl}_{2}$ treatment. For $\mathrm{HgCl}_{2}$ treatment, $50 \mu \mathrm{L} \mathrm{of} \mathrm{HgCl}_{2}$ 
in methanol $(10 \mathrm{mM})$ solution are dropped onto $\mathrm{HgTe}$ film and spin-dried after $15 \mathrm{~s}$. Then the film is rinsed with IPA. This procedure is repeated twice. Finally, an EDT ligand-exchange is performed by dipping the film in a solution of EDT in acetonitrile at $1 \%$ (in volume) for $30 \mathrm{~s}$ and rinsing with pure acetonitrile. A $80 \mathrm{~nm}$ Au top electrode is deposited with thermal evaporation though a shadow mask under a vacuum of $\approx 5 \times 10^{-6} \mathrm{mbar}$ at the rate of $3 \AA / \mathrm{s}$. The thickness was monitored with in situ quartz crystals. The substrate holder was rotated during the deposition to ensure an homogeneous thickness. The overlap area of both electrodes FTO and Au defined as the device area of $1 \mathrm{~mm}^{2}$.

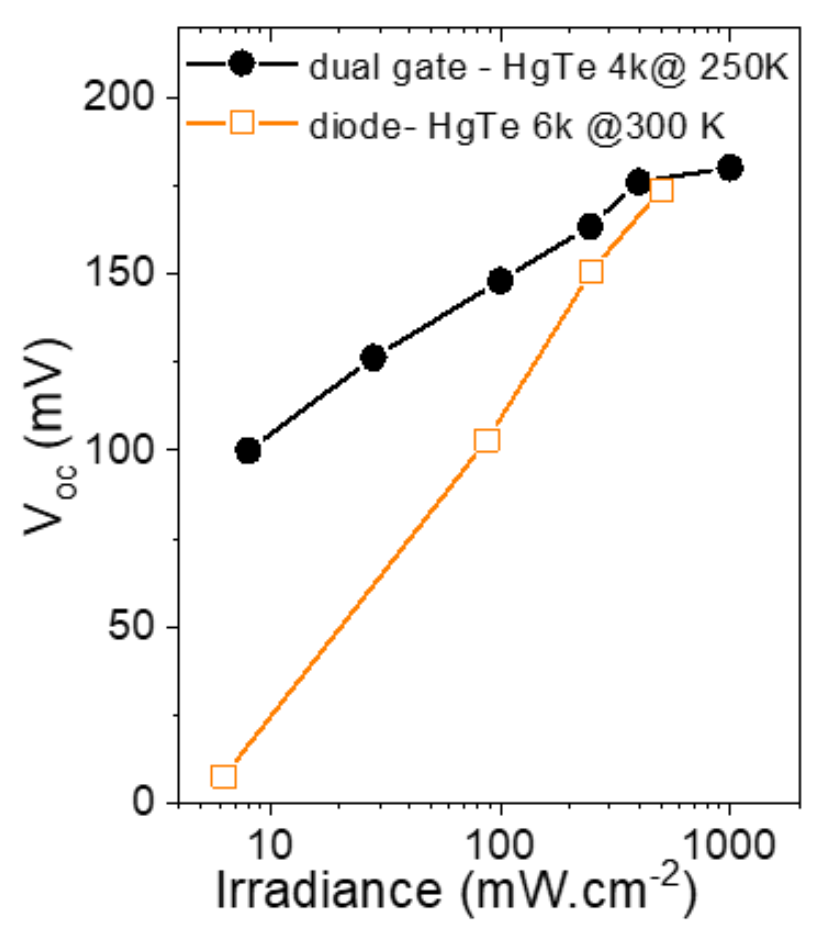

Figure $S$ 19: Open circuit voltage as a function of the light irradiance $(\lambda=1.55 \mu \mathrm{m})$ while the device is operated in the $n-p\left(V_{G 1}=4 V ; V_{G 2}=-4 \mathrm{~V}\right)$. The orange curve is the one obtained from a diode whose structure is made of ITO/HgTe 6k/Ag $2 \mathrm{Te} / \mathrm{Au}$, where $\mathrm{HgTe} 6 \mathrm{k}$ stands for $\mathrm{HgTe} C Q D$ s with a bandgap of $800 \mathrm{meV}\left(6000 \mathrm{~cm}^{-1}\right)$. 


\section{Polydisperse materials}

Figure S 20 provides TEM images of the two HgTe CQD populations and the mixture used to generate a polydisperse population. Figure $S 21$ a shows the absorption spectra of the two populations of $\mathrm{HgTe}$ tripods shown in Figure $\mathrm{S} \mathrm{20}$, as well as the spectrum obtained after their mixing. Figure $\mathrm{S} 21 \mathrm{~b}$ shows the transfer curves for a transistor made of only one population of tripod and for the film obtained by mixing two populations. We observe a reduced current modulation while polydispersity gets higher. Last, we follow the open circuit voltage for a film of made of two populations of tripods deposited on the dual-gate device. Maximum $V_{o c}$ values are reduced by a factor 3 compared to the monodisperse film (figure 3 of main text)

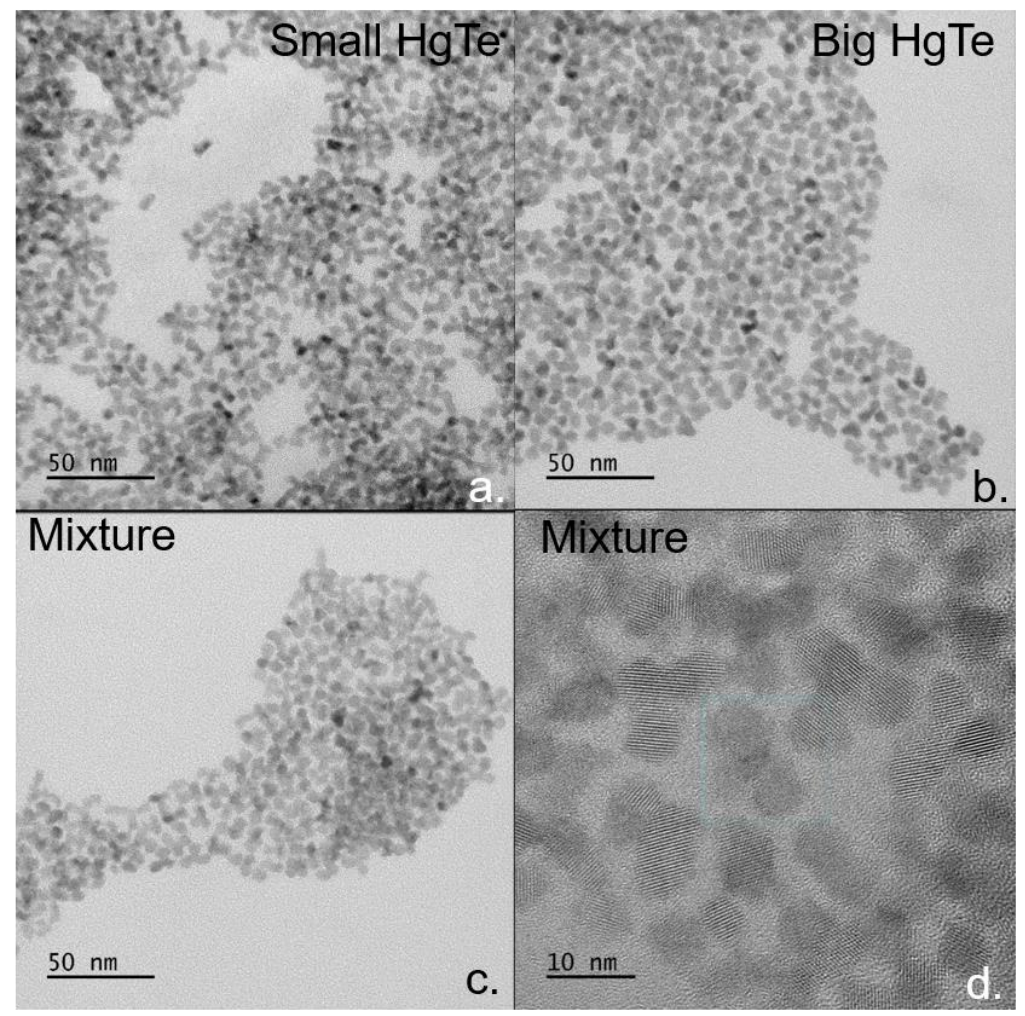

Figure S 20: $a, b$ and $c$ are respectively TEM images of the small, large and the mixture of HgTe $C Q D$ s used to test the impact of polydispservity of the transport and phototransport. D. High resolution picture on the mixture sample.
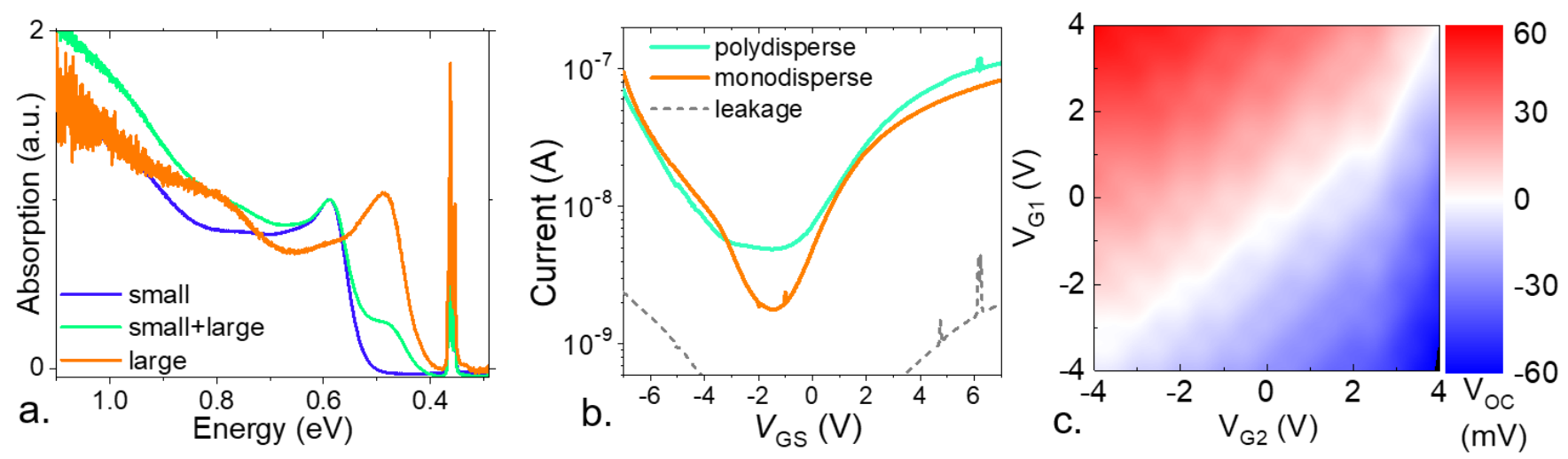

Figure S 21: a. Infrared absorption spectra of two populations of HgTe CQDs with a cut-off wavelength around $4000 \mathrm{~cm}^{-1}(\approx 0.5 \mathrm{eV})$ and their mixture. b. Transfer curves in cases of monodisperse and polydisperse HgTe CQD films, also see Figure S10. c. Open circuit voltage map as a function of applied gate 1 and gate 2 biases for a polydisperse film. 


\section{Quasi-spherical HgTe nanocrystals}

Quasi-spherical HgTe nanocrystals synthesis: The procedure is taken from ref 23 . In a $100 \mathrm{~mL}$ three neck flask, $36 \mathrm{~mL}$ of oleylamine are degassed under vacuum and heated to $110^{\circ} \mathrm{C}$ for $1 \mathrm{~h}$. Meanwhile, $288 \mathrm{mg}$ of $\mathrm{HgBr}_{2}(0.8 \mathrm{mmol})$ are dissolved in $3.6 \mathrm{~mL}$ of oleylamine under sonication. Then, after degazing the solution, $0.4 \mathrm{~mL}$ of TOP:Te $(1 \mathrm{M})$ are added $(t=0)$ and kept in a vial. The mixture is transferred in a syringe just before addition. After mixing 10 min under $\mathrm{N}_{2}$ and at $110{ }^{\circ} \mathrm{C}$, the mixture is quickly injected in the OLA flask. After $20 \mathrm{~s}, 3 \mathrm{~mL}$ of $10 \%$ (in volume) DDT in toluene is injected and a water bath is used to decrease quickly the temperature. The content of the flask is transferred into 2 centrifuge tubes and $\mathrm{MeOH}$ was added. After centrifugation the formed pellets are redispersed in toluene. The solution is precipitated a second time with absolute $\mathrm{EtOH}$. Again, the formed pellet is redispersed in toluene. At this step the nanocrystals are centrifuged in pure toluene to get rid of the lamellar phase. The solid phase is discarded. The solution is precipitated one last time with $\mathrm{MeOH}$ before centrifugation and drying under vacuum. It is then redispersed in $2 \mathrm{~mL}$ toluene. The obtained particles are non- branched and present a quasi-spherical shape according to TEM, see Figure $S$ 22a-b and Figure $S 23$ for STEM HAADF. The absorption spectrum is given in Figure $S$ 22c.
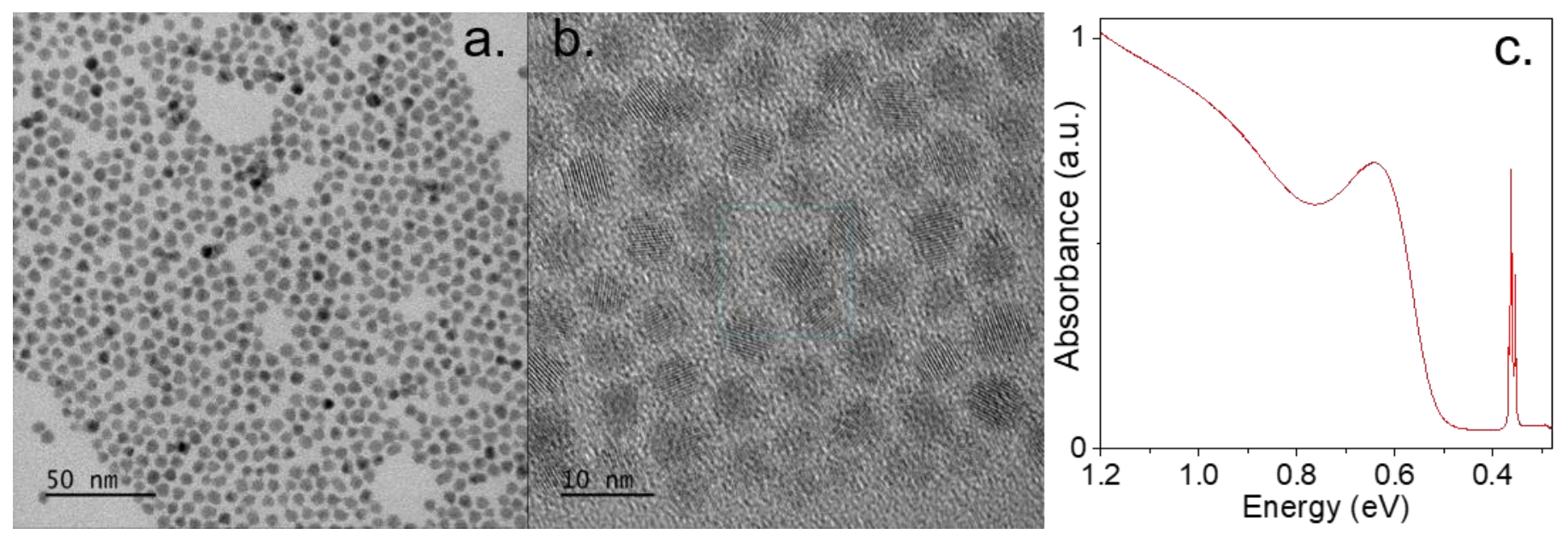

Figure S 22: a. TEM of quasi-spherical HgTe CQDs. b. High resolution TEM of quasi-spherical HgTe CQDs. c. Absorption spectrum of HgTe nanocrystals with quasi-spherical shape.

STEM-HAADF high resolution imaging shows that quasi-sphere shape CQDs nevertheless express some clear facets. 


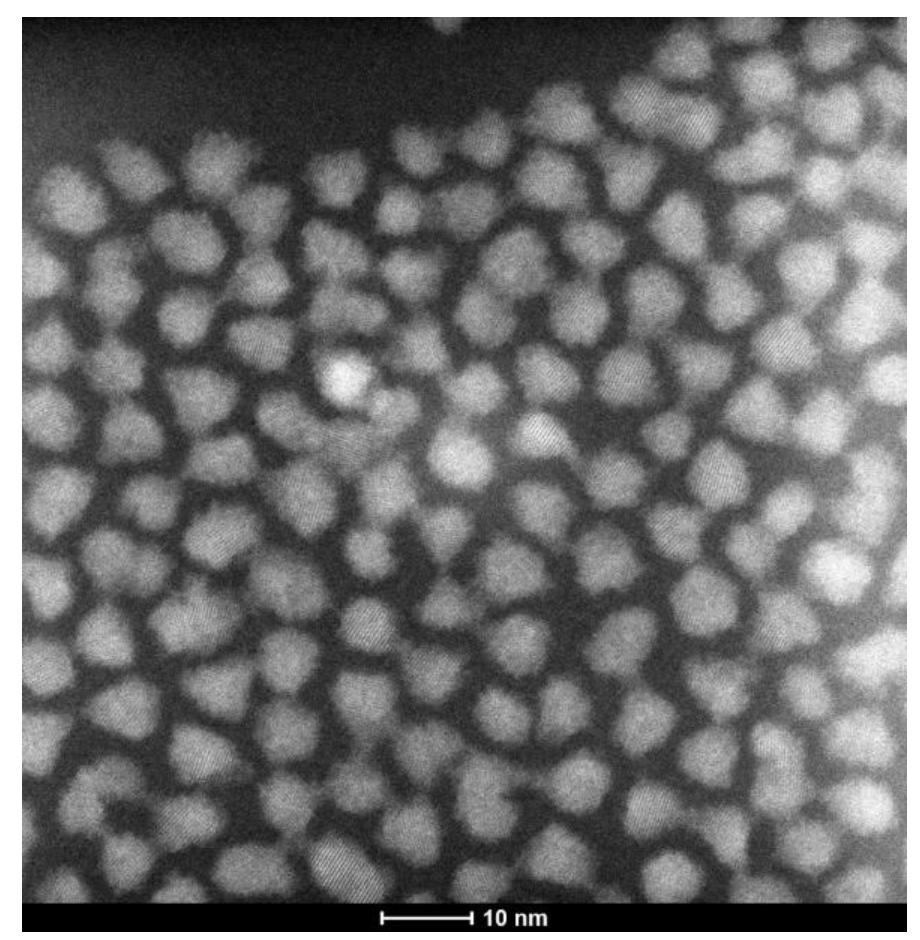

Figure S 23: STEM HAADF image of quasi spherical HgTe CQDs.

EDX mapping of the quasi spherical HgTe NCs, confirms that, as for tripod shape particles, no obvious surface oxidation is observed. On the other hand, the $\mathrm{Hg}$ to Te ratio appears to be different from the one observed for tripods (Te atomic $\approx 55 \%$ for tripods and $60-65 \%$ for quasi spherical CQDs). As it may have been anticipated the change of surface shape also affect the relative final atomic ratio and consequently the doping of the particles. Presence of uncoordinated Te on the surface ${ }^{24}$ was already pointed to lead to trap states near by the valance band and this effect appears to be stronger for sphere shape particles compared to tripods. 


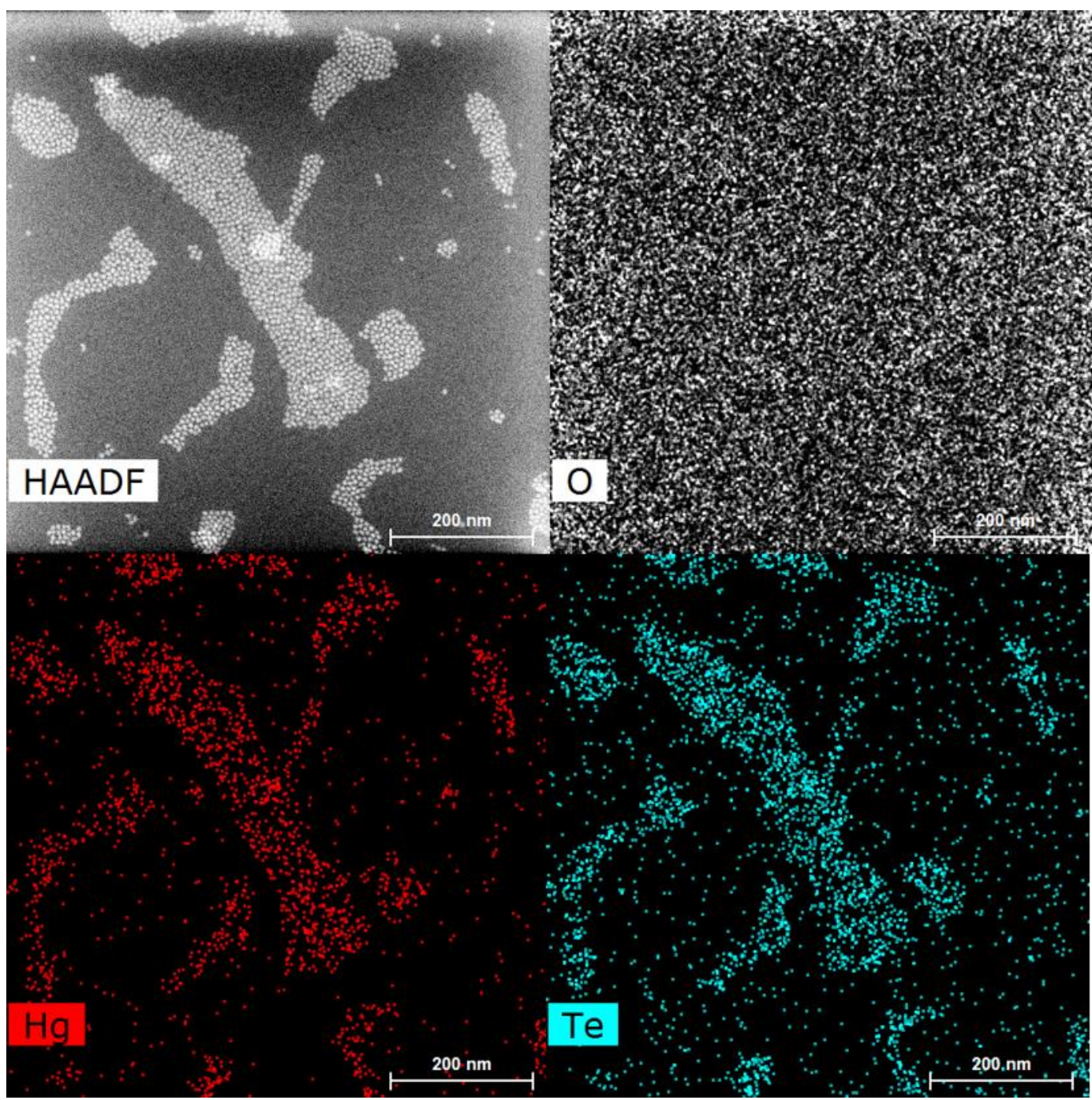

Figure S 24: STEM HAADF and EDX mapping of quasi spherical $\mathrm{HgTe} C Q D$ s for $\mathrm{Hg}, \mathrm{Te}$ and $\mathrm{O}$ atoms.

Electrodes fabrication for electrolyte gated transistor: A polished $\mathrm{Si} / \mathrm{SiO}_{2}$ substrate is first rinsed and then sonicated in acetone for $5 \mathrm{~min}$. The wafer is then rinsed with acetone and isopropanol, dried with a $\mathrm{N}_{2}$ flow and finally cleaned with an $\mathrm{O}_{2}$ plasma for $5 \mathrm{~min}$. Then, AZ 5214E resist is spin coated onto the substrate and baked at $110{ }^{\circ} \mathrm{C}$ for $90 \mathrm{~s}$. The resist is exposed through a shadow mask to UV illumination for $2 \mathrm{~s}$. The resist is baked again at $125^{\circ} \mathrm{C}$ for $2 \mathrm{~min}$ and re-exposed to UV (without the mask) for $40 \mathrm{~s}$. The resist is then developed using AZ 726 for $25 \mathrm{~s}$. The film was rinsed with water and dried. The remaining organic layer is removed by an $\mathrm{O}_{2}$ plasma cleaning. $5 \mathrm{~nm}$ of chromium and $80 \mathrm{~nm}$ of gold are evaporated, and the remaining resist is removed by immersing the film in acetone for one hour. The electrodes are then rinsed with isopropanol and finally dried. The interdigitated electrodes include 25 pairs of digits, each $2.5 \mathrm{~mm}$ long with a $20-\mu \mathrm{m}$ gap between them. The optical area of the device is $0.037 \mathrm{~cm}^{2}$.

Electrolyte gating: For electrolyte gating we first mix in a glove box $0.5 \mathrm{~g}$ of $\mathrm{LiClO}_{4}$ with $2.3 \mathrm{~g}$ of PEG $\left(\mathrm{Mw}=10 \mathrm{~kg} \cdot \mathrm{mol}^{-1}\right)$. The vial is then heated at $170^{\circ} \mathrm{C}$ on a hot plate for $2 \mathrm{~h}$ until the solution turned clear. The electrolyte solution is warmed to around $100{ }^{\circ} \mathrm{C}$ and brushed on the top of the CQD film. 
Large dynamics absorption spectrum is a strategy to reveal sub band gap states. In particular the magnitude of the Urbach energy (Eu) is obtained by fitting the sub band-gap absorption with an exponential decay term ${ }^{25}: \exp (-E / E u)$. To avoid the scattering contribution often observed in the absorption spectrum when conducting measurement in transmission configuration, we choose to rather measure the photocurrent spectrum. The latter is free of any contribution from scattering or from absorption coming from the ligand. The spectra for quasi sphere and tripodic shaped $\mathrm{HgTe}$ CQDs are provided in Figure S 25a. Urbach energy for the two materials are close and around 20 meV. A low optical cross section associated with the trap state may explain the lack of difference observed for the two shapes of particles.
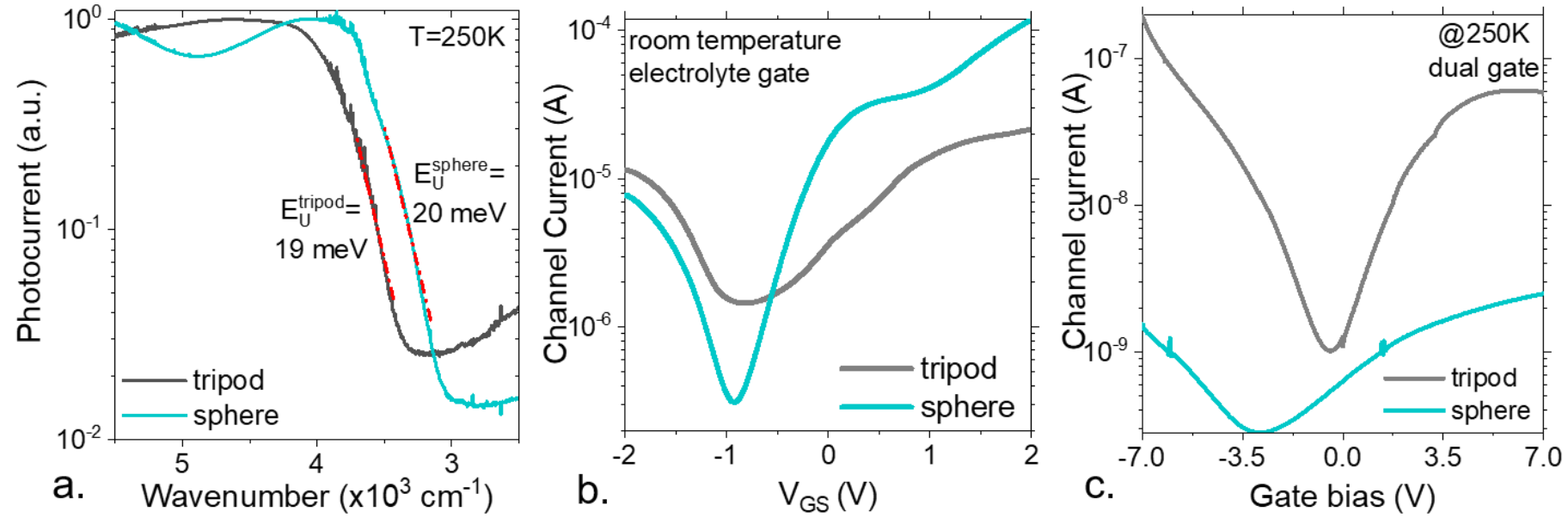

Figure S 25: a. Large dynamic photocurrent spectra for quasi spherical and tripodic shape HgTe CQDs. Photocurrent spectra are acquired at $250 \mathrm{~K}$. b. Transfer curves (drain current as a function of applied gate voltage) for a channel made of quasi spherical and tripodic shape HgTe CQDs while the gate is made of an electrolyte to achieve room temperature gating. Part $b$ is adapted from ref 23 Copyright (2021) American Chemical Society. c. Transfer curves (drain current as a function of applied gate voltage) for a channel made of quasi spherical and tripodic shape HgTe CQDs while maintaining the dual gate configuration with $\mathrm{Al}_{2} \mathrm{O}_{3}$ gate dielectric layer, described in Figure 1 and operated at $250 \mathrm{~K}$.

Using electrolyte, it is possible to obtain gate effect in this $\mathrm{HgTe}$ CQD thin films at room temperature. In this configuration, the spheres present the largest current modulation (on/off ratio>100), while the tripods only show a 1 order of magnitude tunability of the channel current, see Figure S 25b. The mobility of the spheres is estimated to be 3 times larger than the one observed for the tripods. At room temperature, traps play a limited role since thermal energy is sufficient to release trapped carriers. Thus, the higher mobility observed for the sphere reflects a better particle packing obtained for this type of particles. On the other hand, at low temperature, we observe a stronger current modulation in the case of the tripods, Figure S 25c. It reflects that when temperature is reduced the effective carrier mobility gets directly impacted by the charge trapping. The stronger nonstoichiometry of the spheres (i.e. excess of Te) generates a higher trap density and the latter reduces the carrier mobility at low temperature. 


\section{REFERENCES}

(1) Gréboval, C.; Chu, A.; Goubet, N.; Livache, C.; Ithurria, S.; Lhuillier, E. Mercury Chalcogenide Quantum Dots: Material Perspective for Device Integration. Chem. Rev. 2021.

(2) Moon, T. K. The Expectation-Maximization Algorithm. IEEE Signal Process. Mag. 1996, 13, 47-60.

(3) van Aarle, W.; Palenstijn, W. J.; De Beenhouwer, J.; Altantzis, T.; Bals, S.; Batenburg, K. J.; Sijbers, J. The ASTRA Toolbox: A Platform for Advanced Algorithm Development in Electron Tomography. Ultramicroscopy 2015, 157, 35-47.

(4) Allan, G.; Delerue, C. Tight-Binding Calculations of the Optical Properties of HgTe Nanocrystals. Phys. Rev. B - Condens. Matter Mater. Phys. 2012, 86, 165437.

(5) Groeneveld, E.; Delerue, C.; Allan, G.; Niquet, Y.-M.; de Mello Donegá, C. Size Dependence of the Exciton Transitions in Colloidal CdTe Quantum Dots. J. Phys. Chem. C 2012, 116, $23160-23167$.

(6) Keuleyan, S. E.; Guyot-Sionnest, P.; Delerue, C.; Allan, G. Mercury Telluride Colloidal Quantum Dots: Electronic Structure, Size-Dependent Spectra, and Photocurrent Detection up to $12 \mu \mathrm{m}$. ACS Nano 2014, 8, 8676-8682.

(7) Hudson, M. H.; Chen, M.; Kamysbayev, V.; Janke, E. M.; Lan, X.; Allan, G.; Delerue, C.; Lee, B.; Guyot-Sionnest, P.; Talapin, D. V. Conduction Band Fine Structure in Colloidal HgTe Quantum Dots. ACS Nano 2018, 12, 9397-9404.

(8) Lhuillier, E.; Keuleyan, S.; Zolotavin, P.; Guyot-Sionnest, P. Mid-Infrared HgTe/As $2 \mathrm{~S}_{3}$ Field Effect Transistors and Photodetectors. Adv. Mater. 2013, 25, 137-141.

(9) Tress, W.; Yavari, M.; Domanski, K.; Yadav, P.; Niesen, B.; Baena, J. P. C.; Hagfeldt, A.; Graetzel, M. Interpretation and Evolution of Open-Circuit Voltage, Recombination, Ideality Factor and Subgap Defect States during Reversible Light-Soaking and Irreversible Degradation of Perovskite Solar Cells. Energy Environ. Sci. 2018, 11, 151-165.

(10) Chu, A.; Gréboval, C.; Prado, Y.; Majjad, H.; Delerue, C.; Dayen, J.-F.; Vincent, G.; Lhuillier, E. Infrared Photoconduction at the Diffusion Length Limit in HgTe Nanocrystal Arrays. Nat. Commun. 2021, 12, 1794.

(11) Grotevent, M. J.; Hail, C. U.; Yakunin, S.; Bachmann, D.; Calame, M.; Poulikakos, D.; Kovalenko, M. V.; Shorubalko, I. Colloidal HgTe Quantum Dot/Graphene Phototransistor with a Spectral Sensitivity Beyond $3 \mu \mathrm{m}$. Adv. Sci. 2021, 8, 2003360.

(12) Noumbé, U. N.; Gréboval, C.; Livache, C.; Chu, A.; Majjad, H.; Parra López, L. E.; Mouafo, L. D. N.; Doudin, B.; Berciaud, S.; Chaste, J.; Ouerghi, A.; Lhuillier, E.; Dayen, J.-F. Reconfigurable 2D/0D p-n Graphene/HgTe Nanocrystal Heterostructure for Infrared Detection. ACS Nano 2020.

(13) Gréboval, C.; Chu, A.; Magalhaes, D. V.; Ramade, J.; Qu, J.; Rastogi, P.; Khalili, A.; Chee, S.S.; Aubin, H.; Vincent, G.; Bals, S.; Delerue, C.; Lhuillier, E. Ferroelectric Gating of Narrow Band-Gap Nanocrystal Arrays with Enhanced Light-Matter Coupling. ACS Photonics 2021, 8, 259-268.

(14) Chen, M.; Lu, H.; Abdelazim, N. M.; Zhu, Y.; Wang, Z.; Ren, W.; Kershaw, S. V.; Rogach, A. L.; Zhao, N. Mercury Telluride Quantum Dot Based Phototransistor Enabling High-Sensitivity Room-Temperature Photodetection at $2000 \mathrm{~nm}$. ACS Nano 2017, 11, 5614-5622.

(15) Dong, Y.; Chen, M.; Yiu, W. K.; Zhu, Q.; Zhou, G.; Kershaw, S. V.; Ke, N.; Wong, C. P.; Rogach, A. L.; Zhao, N. Solution Processed Hybrid Polymer: HgTe Quantum Dot Phototransistor with High Sensitivity and Fast Infrared Response up to $2400 \mathrm{~nm}$ at Room Temperature. Adv. Sci. 2020, 7, 2000068.

(16) Martinez, B.; Ramade, J.; Livache, C.; Goubet, N.; Chu, A.; Gréboval, C.; Qu, J.; Watkins, W. L.; Becerra, L.; Dandeu, E.; Fave, J. L.; Méthivier, C.; Lacaze, E.; Lhuillier, E. HgTe Nanocrystal Inks for Extended Short-Wave Infrared Detection. Adv. Opt. Mater. 2019, 7, 1900348.

(17) Tang, X.; Ackerman, M. M.; Shen, G.; Guyot-Sionnest, P. Towards Infrared Electronic Eyes: Flexible Colloidal Quantum Dot Photovoltaic Detectors Enhanced by Resonant Cavity. Small 2019, 15, 1804920. 
(18) Ackerman, M. M.; Chen, M.; Guyot-Sionnest, P. HgTe Colloidal Quantum Dot Photodiodes for Extended Short-Wave Infrared Detection. Appl. Phys. Lett. 2020, 116, 083502.

(19) Rastogi, P.; Chu, A.; Dang, T. H.; Prado, Y.; Gréboval, C.; Qu, J.; Dabard, C.; Khalili, A.; Dandeu, E.; Fix, B.; Xu, X. Z.; Ithurria, S.; Vincent, G.; Gallas, B.; Lhuillier, E. Complex Optical Index of HgTe Nanocrystal Infrared Thin Films and Its Use for Short Wave Infrared Photodiode Design. Adv. Opt. Mater. 2021, 2002066.

(20) Ackerman, M. M.; Tang, X.; Guyot-Sionnest, P. Fast and Sensitive Colloidal Quantum Dot MidWave Infrared Photodetectors. ACS Nano 2018, 12, 7264-7271.

(21) Ackerman, M. M.; Chen, M.; Guyot-Sionnest, P. HgTe Colloidal Quantum Dot Photodiodes for Extended Short-Wave Infrared Detection. Appl. Phys. Lett. 2020, 116, 083502.

(22) Martinez, B.; Ramade, J.; Livache, C.; Goubet, N.; Chu, A.; Gréboval, C.; Qu, J.; Watkins, W. L.; Becerra, L.; Dandeu, E.; Fave, J. L.; Méthivier, C.; Lacaze, E.; Lhuillier, E. HgTe Nanocrystal Inks for Extended Short-Wave Infrared Detection. Adv. Opt. Mater. 2019, 7, 1900348.

(23) Prado, Y.; Qu, J.; Gréboval, C.; Dabard, C.; Rastogi, P.; Chu, A.; Khalili, A.; Xu, X. Z.; Delerue, C.; Ithurria, S.; Lhuillier, E. Seeded Growth of HgTe Nanocrystals for Shape Control and Their Use in Narrow Infrared Electroluminescence. Chem. Mater. 2021, 33, 2054-2061.

(24) Geiregat, P.; Houtepen, A. J.; Sagar, L. K.; Infante, I.; Zapata, F.; Grigel, V.; Allan, G.; Delerue, C.; Van Thourhout, D.; Hens, Z. Continuous-Wave Infrared Optical Gain and Amplified Spontaneous Emission at Ultralow Threshold by Colloidal HgTe Quantum Dots. Nat. Mater. 2018, 17, 35-42.

(25) Guyot-Sionnest, P.; Lhuillier, E.; Liu, H. A Mirage Study of CdSe Colloidal Quantum Dot Films, Urbach Tail, and Surface States. J. Chem. Phys. 2012, 137, 154704. 